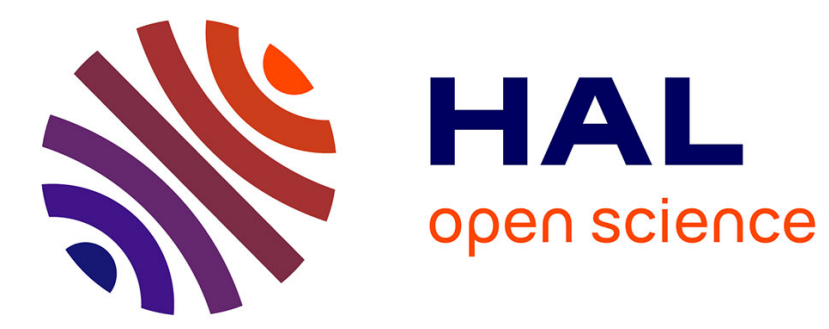

\title{
On Temporal Aggregators and Dynamic Programming
}

\author{
Philippe Bich, Jean-Pierre Drugeon, Lisa Morhaim
}

\section{To cite this version:}

Philippe Bich, Jean-Pierre Drugeon, Lisa Morhaim. On Temporal Aggregators and Dynamic Programming. Economic Theory, 2018, 66, pp.787-817. 10.1007/s00199-017-1045-0 . halshs-01437496

\section{HAL Id: halshs-01437496 \\ https://shs.hal.science/halshs-01437496}

Submitted on 17 Jan 2017

HAL is a multi-disciplinary open access archive for the deposit and dissemination of scientific research documents, whether they are published or not. The documents may come from teaching and research institutions in France or abroad, or from public or private research centers.
L'archive ouverte pluridisciplinaire HAL, est destinée au dépôt et à la diffusion de documents scientifiques de niveau recherche, publiés ou non, émanant des établissements d'enseignement et de recherche français ou étrangers, des laboratoires publics ou privés. 


\title{
On Temporal Aggregators And Dynamic Programming*
}

\author{
Philippe Bich Jean-Pierre Drugeon $^{\ddagger}$ and Lisa Morhaim ${ }^{\S}$
}

Monday $16^{\text {th }}$ January, 2017

\begin{abstract}
This paper proposes dynamic programming tools for payoffs based on aggregating functions that depend on the current action and the future expected payoff. Some regularity properties are provided on the aggregator to establish existence, uniqueness and computation of the solution to the Bellman equation. Our setting allows to encompass and generalize many previous results based upon additive or non-additive payoff functions.
\end{abstract}

Keywords: Dynamic Programming, Temporal Aggregators, Intertemporal Choice. JEL Classification: C61, D90.

*This research was completed thanks to the Novo Tempus research grant, ANR-12BSH1-0007, Program BSH1-2012. The authors would like to thank the participants of conferences on recursive methods held in Glasgow by June 2014 and in Phoenix by March 2015, of the PET conference, Seattle, July 2014, and the ones of the SAET conference, Tokyo, August 2014.

${ }^{\dagger}$ Paris School of Economics and University of Paris 1 Panthéon-Sorbonne.

${ }^{\ddagger}$ Paris School of Economics and Centre National de la Recherche Scientifique.

$\S$ University of Paris 2 Panthéon-Assas, CRED Paris Center for Law and Economics. 
1 INTRODUCTION 1

2 Aggregators Versus ReCURSive Payoffs $\quad 3$

2.1 Aggregators, $A$-payoff and $A$-recursive payoff $\ldots \ldots \ldots$

2.2 Preference relationships on $Z^{\mathbf{N}} \ldots \ldots \ldots \ldots$

3 Optimization PROGRAMS $\quad \mathbf{5}$

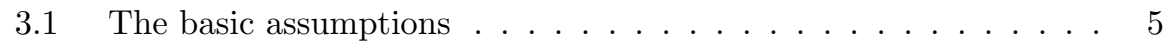

3.2 Definitions of maximization programs . . . . . . . . 6

3.3 Solutions and values of the programs may differ . . . . . . . 7

3.4 A condition for the programs to coincide . . . . . . . 7

4 DYNAMIC PROGRAMMING $\quad \mathbf{8}$

4.1 Existence of a solution to the Bellman equation under uniform continuity property in $v \ldots \ldots \ldots \ldots \ldots$

$4.1 .1 \quad$ Uniform continuity in $v \ldots \ldots \ldots \ldots$

4.1.2 Compact Uniform continuity in $v \ldots \ldots \ldots$

4.1.3 The existence result . . . . . . . . . . . . . . . 13

4.2 Existence, uniqueness and computation of a solution to the Bellman equation under weak continuity . . . . . . . . . 13

$4.2 .1 \quad$ Weak continuity . . . . . . . . . . . . . . 14

4.2.2 Existence, uniqueness and computation of the solution to the Bellman equation . . . . . . . . . . . 15

4.2 .3 On Theorems 4.1 and $4.2 \ldots \ldots \ldots \ldots$

4.2.4 Additive aggregators and biconvergent aggregators . . . 16

$\begin{array}{lll}\text { A Proof of Proposition 2.1. } & \mathbf{1 8}\end{array}$

$\begin{array}{lll}\text { B Proof of Proposition 3.1. } & 19\end{array}$

$\begin{array}{lrl}\text { C } & \text { Proof of Proposition 3.2. } & \mathbf{2 1}\end{array}$

$\begin{array}{lll}\text { D Proof of Lemma } 4.1 & \mathbf{2 1}\end{array}$

E $\quad$ Proof of Proposition 4.1. 22

F $\quad$ Proof of Proposition 4.2. 23

G Proof of Theorem 4.1. 24

H $\quad$ Proof of Proposition $4.3 \quad \mathbf{2 7}$

$\begin{array}{lrr}\text { I } & \text { Proof of Proposition } 4.4 & \mathbf{2 8}\end{array}$

$\begin{array}{lrr}\text { J } & \text { Proof of Theorem } 4.2 & \mathbf{2 9}\end{array}$

K Another Definition of Biconvergence 33

$\begin{array}{lrrrl}\text { L } & \text { Proof of Corollary } 4.1 & 34\end{array}$ 


\section{INTRODUCTION}

Dating from Stokey Lucas and Prescott [18], dynamic programming techniques and the Bellman principle of optimality have been developed to analyze intertemporal equilibria of infinite horizon problems through sequences of two-period problems. In particular, it involves studying existence, uniqueness and computation of the solutions to the Bellman equation.

In the analyzis of intertemporal equilibria, the notion of recursive intertemporal payoff have played a central role. Consider an agent who takes some decision $z_{t}$ in some action set $Z$ at each date $t \in \mathbf{N}$. An intertemporal payoff function associates to every action stream $\underset{\sim}{z}=\left(z_{t}\right)_{t \in \mathbf{N}}$ in $Z^{\mathbf{N}}$ some payoff $U(z)$, so that the preferences of the agent, defined on $Z^{\mathbf{N}}$, are represented by $U$. The function $U$ is recursive if the intertemporal payoff from today $U(z)=U\left(z_{0}, z_{1}, \ldots, z_{n}, \ldots\right)$, is a function $A\left(z_{0}, U\left(z_{1}, \ldots, z_{n}, \ldots\right)\right)$ of the action today $z_{0}$ and the intertemporal payoff from tomorrow $U\left(z_{1}, \ldots, z_{n}, \ldots\right)$. The function $A$ involved in this definition aggregates the current action $z$ and the future payoff into the current payoff, and is called aggregating function, or aggregator.

There are two main approaches to deal with recursive payoffs: the first, initiated by Koopmans ${ }^{1}[7]$, provides an axiomatization of preferences which leads to a recursive payoff function. The second takes the aggregator function as a primitive, and provides conditions under which there exists some recursive payoff (see Stokey and Lucas [17], Becker and Boyd [2], Streufert [20], Le Van and Vailakis [9]). ${ }^{2}$

In this paper, we propose some dynamic programming tools without any reference to recursive payoffs. Two main arguments advocates for such an approach. First, such a recursive payoff does not always exist. Second, as proved in this paper, even if such a recursive payoff exists, preorders on the action sequences induced by a given aggregator and by an associated recursive payoff may be different.

This setting allows also to encompass models with possibly unbounded pay-

\footnotetext{
${ }^{1}$ This approach is presented in Becker and Boyd (see Section 3.3.1. in [2]).

${ }^{2}$ In Yao [22], the aggregator and the recursive payoff are taken together as primitives.
} 
offs. The use of dynamic programming tools for additively separable models with unbounded payoffs was first initiated by Boyd [3], Alvarez and Stokey [1], Duran [4] and Le Van and Morhaim [8].

Four distinct approaches have been proposed to deal with Bellman equation. First, the Banach contraction theorem (or local version of it) can be applied: for the bounded case, see Stokey Lucas and Prescott [18], and for the possibly unbounded case, see Rincon-Zapatero and Rodriguez-Palmero [14] or Martins-da-Rocha and Vailakis [11]. Second, a direct approach can be used, and requires some Lipschitz condition on the aggregator (see Le Van and Morhaim or Le Van and Vailakis [9]). Third, Streufert has proposed to replace the Lipschitz condition by some topological property (biconvergence) on the recursive payoff function and the feasibility set (Streufert [19, 20]). Last, an order-theoretic fixed-point machinery can also be used to replace the Banach fixed-point theorem (see Kamihigashi [6]).

All these approaches are sometimes connected, and each one has its advantages from some particular point of view: for example, the approach proposed by Kamihigashi [6] avoids topological assumptions, and encompasses the existence and uniqueness part of Rincon-Zapatero and Rodriguez-Palmero [14] and Martins-da-Rocha and Vailakis [11]'s work. But these approaches only consider additive separable models, and they require assumptions that may be difficult to check. Streufert's biconvergence assumption is singular in the literature in the sense that it does not imply nor is implied by the other results.

Our contribution proposes to unify most of these papers (e.g., Streufert [19, 20], Kamihigashi [7] or Le Van and Vailakis [9]). In particular, it encompasses some aggregators studied in the literature such as the ones in Jaśkiewicz, Matkowski and Nowak $[5]^{3}$, and Blackwell and Thompson aggregators (see Marinacci Montrucchio [10]). This is done through a weak continuity assumption on the aggregator. Together with some transversality condition, this ensures the existence and the uniqueness of a fixed-point for the Bellman operator, and also provides an algorithm to reach the value

\footnotetext{
${ }^{3}$ They consider an additive model where the constant discount rate is repaced by a function.
} 
function starting from a suitable initial function.

This article is organized as follows. Section 2 introduces aggregators and recursive payoffs, and explains why these payoffs could induce different preferences. Section 3 introduces the aggregator-based optimization program, and compares it to the optimization program associated to any recursive payoff (when it exists). Section 4 establishes general conditions for the value function of the aggregator-based optimization problems to be the unique fixed point of the Bellman operator, allowing this value function to be reached by an algorithm. The main proofs are given in Appendix.

\section{AgGregators VERSUS RECURSIVE PAYOFFS}

\subsection{Aggregators, $A$-Payoff and $A$-recursive payoff}

Time is discrete. Consider an entity (agent, firm, player, decision maker,....) that chooses an action $z_{t}$ at each date $t \in \mathbf{N}$ (the set of nonnegative integers) in some action set $Z$. Preferences on $Z^{\mathbf{N}}$ are usually modelized through a utility function on $Z^{\mathbf{N}}$. In this paper, an aggregator is instead taken as a primitive:

Definition 2.1. A (temporal) aggregator is a mapping

$$
A: Z \times[-\infty,+\infty] \rightarrow[-\infty,+\infty]
$$

where $Z$ is called an action set. ${ }^{4}$

For every current action $z_{0}$ and every future payoff $v \in[-\infty,+\infty], A\left(z_{0}, v\right)$ aggregates the current action $z_{0}$ and the future payoff $v$ into one current payoff. Similarly, given two actions $z_{0}$ and $z_{1}$ at $t=0$ and $t=1$, and given a future payoff $v \in[-\infty,+\infty]$ at date $t=2$, the aggregate payoff at $t=0$ can be written $A\left(z_{0}, A\left(z_{1}, v\right)\right)$. Iterating, for every sequence $z=\left(z_{t}\right)_{t \in \mathbf{N}}$ in

\footnotetext{
${ }^{4}$ In the existing literature, the aggregator $A$ is generally defined on $Z \times \mathbf{R}$. In this case, if $A$ is increasing with respect to its second argument (an assumption that is made in this paper), such aggregator can be extended to a function $Z \times \overline{\mathbf{R}} \rightarrow \overline{\mathbf{R}}$ by letting $A(z,+\infty)=\lim _{v \rightarrow+\infty} A(z, v)$ and $A(z,-\infty)=\lim _{v \rightarrow-\infty} A(z, v)$.
} 
$Z^{\mathbf{N}}$, every $T \in \mathbf{N}^{*}$ (the set of positive integers) and every $v \in[-\infty,+\infty]$, we define

$$
A^{T}(z, v)=A\left(z_{0}, A\left(z_{1}, A\left(z_{2}, \ldots, A\left(z_{T-2}, A\left(z_{T-1}, v\right)\right) \cdots\right)\right)\right)
$$

as the aggregate payoff at $t=0$, given actions $z_{0}, \ldots, z_{T-1}$ of the entity at dates $t=0, \ldots, T-1$ and a future payoff $v$ at date $t=T$.

From now on, we consider that $A$ is a given aggregator.

Remark 2.1. (Additive case) If $A(z, v)=u(z)+\beta v$ for some instantaneous utility function $u(\cdot)$ and some discount factor $\beta \in] 0,1[$, then the intertemporal payoff can be written, when it exists and for every sequence of actions over time $\underset{z}{z}=\left(z_{t}\right)_{t \in \mathbf{N}}$, as follows:

$$
\sum_{t=0}^{+\infty} \beta^{t} u\left(z_{t}\right)=\lim _{T \rightarrow+\infty} \sum_{t=0}^{T} \beta^{t} u\left(z_{t}\right)=\lim _{T \rightarrow+\infty} A^{T}(z, 0) .
$$

When such a limit does not exist, one could consider instead the supremum limit (optimistic point of view), or the infimum limit (pessimistic point of view). This motivates the following definition.

Definition 2.2. Given a sequence of actions $\underset{\sim}{z}$ in $Z^{\mathbf{N}}$ :

(i) the $A$-payoff (when it exists) is $w(z)=\lim _{T \rightarrow+\infty} A^{T}(\underset{z}{z}, 0)$.

(ii) the upper $A$-payoff is $\bar{w}(z)=\varlimsup_{\sim} \rightarrow+\infty A^{T}(\underset{z}{z}, 0)$.

(iii) the lower $A$-payoff is $\underline{w}(z)=\underline{\lim }_{T \rightarrow+\infty} A^{T}(z, 0)$.

Given an aggregator, another way to define a payoff is to consider a recursive utility function associated to the aggregator $A$, which we now define. In the following, let the shift operator $S: Z^{\mathbf{N}} \rightarrow Z^{\mathbf{N}}$ be defined by $S\left(z_{0}, z_{1}, \ldots\right)=$ $\left(z_{1}, z_{2}, \ldots\right)$.

Definition 2.3. Given an aggregator $A: Z \times[-\infty,+\infty] \rightarrow[-\infty,+\infty]$, a payoff function $U: Z^{\mathbf{N}} \rightarrow[-\infty,+\infty]$ is said to be $A$-recursive if

$$
\forall z \in Z^{\mathbf{N}}, U(z)=A\left(z_{0}, U(S(z))\right)
$$

Remark that if $A \geq 0$, then upper $A$-payoff, lower $A$-payoff and $A$-payoff coincide: indeed, this assumption implies that the sequence $\left(A^{T}(z, 0)\right)_{T \in \mathbf{N}}$ is non decreasing, thus it converges (in $[-\infty,+\infty]$ ). 


\subsection{PREFERENCE RELATIONShips ON $Z^{\mathbf{N}}$}

Consider an $A$-recursive payoff $U$, the $A$-payoffs $w, \underline{w}$ and $\bar{w}$ defined in Section 2.1. Each of these four functions defines a preference on some subset of $Z^{\mathbf{N}}$ as follows:

We first associate to $U$ the pre-order $\precsim_{U}$ on $Z^{\mathbf{N}}$ defined by:

$$
\forall\left(z, z^{\prime}\right) \in Z^{\mathbf{N}} \times Z^{\mathbf{N}}, \underset{z}{z} z^{\prime} \Leftrightarrow U(z) \leq U\left(z^{\prime}\right)
$$

Second, if $\underset{\sim}{Z^{\text {conv }}}$ denotes the set of sequences $\underset{\sim}{z} Z^{\mathbf{N}}$ such that the sequence $\left(A^{n}(z, 0)\right)_{n \in \mathbf{N}}$ converges in $[-\infty,+\infty]$, we can associate to $w$ the pre-order $\precsim_{w}$ on $Z^{\text {conv }}$ defined by

$$
\forall\left(\underset{\sim}{z}, z^{\prime}\right) \in \underset{\sim}{Z^{\mathrm{conv}}} \times{\underset{\sim}{Z}}^{\mathrm{conv}}, \underset{\sim}{z} \precsim_{w} z^{\prime} \Leftrightarrow w\left(\underset{\sim}{z} \leq \leq w\left(z^{\prime}\right)\right.
$$

the pre-orders $\precsim_{\underline{w}}$, $\precsim_{\bar{w}}$ being defined similarly on $Z^{\mathbf{N}}$.

The preferences induced by a given aggregator $A$, and those induced by any $A$-recursive payoff can be different (for the proof, see the appendix):

Proposition 2.1. There exists an aggregator $A$ such that for every A-recursive payoff $U$, the pre-order $\precsim_{A}$ (induced by the aggregator $A$ on $Z^{\mathrm{conv}}$ ) and the pre-order $\precsim_{U}$ (induced by some A-recursive payoff function $U$ associated to A) differ.

\section{Optimization PROGRAMS}

\subsection{The BASIC ASSUMPtions}

The two following assumptions will be retained through the whole exposition:

Assumption IM. [Increasing Monotonicity]

$\forall z \in Z, v \rightarrow A(z, v)$ is non-decreasing.

Assumption B. [Boundary]

For every $z \in Z, \lim _{v \rightarrow-\infty} A(z, v)=A(z,-\infty)$ and $\lim _{v \rightarrow+\infty} A(z, v)=$ $A(z,+\infty)$.

Convention I. $-[$ Infinity $]+\infty-(+\infty)=0$ and $-\infty-(-\infty)=0$. 


\subsection{Definitions OF MAXIMIZATION PROGRAMS}

Let $X$ be the set of states, and $x_{t} \in X$ the state at time $t$.

The law of evolution of $x_{t}$ is defined through a multivalued mapping $\Gamma$, defined from $X$ to $X$, with nonempty values, such that $x_{t+1} \in \Gamma\left(x_{t}\right)$. Given two states $x_{t}$ and $x_{t+1}, \Omega\left(x_{t}, x_{t+1}\right)$ is the set of feasible actions at time $t$, where $\Omega$ is a multivalued mapping defined from $\operatorname{Gr}(\Gamma)$ - the graph of $\Gamma$ - to $Z$ with nonempty values.

Given an initial state $x_{0}$ in $X, \Sigma\left(x_{0}\right)$ is the set of sequences of actions that are feasible from $x_{0}$, that is:

$$
\begin{array}{r}
\Sigma\left(x_{0}\right)=\left\{\left(z_{t}\right)_{t \in \mathbf{N}} \in Z^{\mathbf{N}}: \exists\left(x_{t}\right)_{t \in \mathbf{N}^{*}} \in X^{\mathbf{N}}: \forall t \in \mathbf{N}, x_{t+1} \in \Gamma\left(x_{t}\right),\right. \\
\left.z_{t} \in \Omega\left(x_{t}, x_{t+1}\right)\right\},
\end{array}
$$

while $\widetilde{\Sigma}\left(x_{0}\right)$ specifies the sequences of actions and states that are feasible from $x_{0}$, that is:

$$
\begin{aligned}
& \widetilde{\Sigma}\left(x_{0}\right)=\left\{\left(\left(z_{t}\right)_{t \in \mathbf{N}},\left(x_{t}\right)_{t \in \mathbf{N}^{*}}\right) \in Z^{\mathbf{N}} \times X^{\mathbf{N}}: \forall t \in \mathbf{N}, x_{t+1} \in \Gamma\left(x_{t}\right),\right. \\
& \left.z_{t} \in \Omega\left(x_{t}, x_{t+1}\right)\right\} \text {. }
\end{aligned}
$$

A model is defined as a triple $(A, \Gamma, \Omega), A$ being an aggregator, and $\Gamma$ and $\Omega$ some feasibility correspondences. Given a model $(A, \Gamma, \Omega)$, an $A$-recursive payoff function $U: Z^{\mathbf{N}} \rightarrow[-\infty,+\infty]$, and $x_{0}$ in $X$, let us consider the following optimization problems:

$$
\sup _{z \in \Sigma\left(x_{0}\right)} \bar{w}(z)
$$

() $\sup _{z \in \Sigma\left(x_{0}\right)} \underline{w}(z)$

(P) $\sup _{z \in \Sigma\left(x_{0}\right)} U(z)$.

Let $\bar{v}^{*}, \underline{v}^{*}$ and $v^{* *}$ defined from $X$ to $[-\infty,+\infty]$ be the value functions respectively of problem $(\bar{P}),(\underline{P})$ and $(P)$.

We are then interested in comparing these programs (i.e. their values and solutions). 


\subsection{SOlutions AND VAlues of THE PROGRAMS MAY DIF- FER}

The following assumption will facilitate the comparison between the programs.

Null Assumption. [Null Consumption] There exists $(\underline{a}, \underline{a}, \ldots, \underline{a}, \ldots) \in \cap_{x \in X} \Sigma(x)$ with $U(\underline{a}, \underline{a}, \ldots, \underline{a}, \ldots)=0$.

Remark 3.1. This assumption is rather weak. This is equivalent to Assumption B6 in Streufert [19]. It could be perceived as a free disposal assumption and is generally valid in economic setups.

The following statement compares the optimization programs defined above (for the proof, see the appendix ).

Proposition 3.1. Let $x_{0} \in X$. Consider the optimization programs $(\bar{P})$, $(\underline{P})$ and $(P)$ :

1. The optimization problems $(\bar{P})$ and $(P)$ (and similarly $(\underline{P})$ and $(P)$ ) may have different solutions.

2. Under Null Assumption, $v^{* *}\left(x_{0}\right) \geq \bar{v}^{*}\left(x_{0}\right)$, and this inequality can be strict.

\subsection{A CONDITION FOR THE PROGRAMS TO COINCIDE}

For every $x_{0} \in X$, define the sets

$$
\Sigma^{0}\left(x_{0}\right)=\left\{z \in \Sigma\left(x_{0}\right): \bar{w}(\underset{\sim}{z})>-\infty\right\}
$$

and

$$
\widetilde{\Sigma}^{0}\left(x_{0}\right)=\left\{\left(\left(z_{t}\right)_{t \in \mathbf{N}},\left(x_{t}\right)_{t \in \mathbf{N}^{*}}\right) \in \widetilde{\Sigma}\left(x_{0}\right): \bar{w}(z)>-\infty\right\}
$$

Definition 3.1. A pair of functions $\left(f_{1}, f_{2}\right) \in V^{2}$, where $V=\mathscr{F}(X,[-\infty,+\infty])$ is the set of functions from $X$ to $[-\infty,+\infty]$, is said to satisfy Transversality if

T1 $\forall x_{0} \in X, \forall(\underset{\sim}{z}, \underset{\sim}{x}) \in \widetilde{\Sigma}^{0}\left(x_{0}\right), \underline{\lim }_{T \rightarrow+\infty}\left(A^{T}\left(\underset{\sim}{z}, f_{1}\left(x_{T}\right)\right)-A^{T}(\underset{\sim}{z}, 0)\right) \geq 0$. 
T2 $\forall x_{0} \in X, \forall(\underset{\sim}{z} \underset{\sim}{x}) \in \widetilde{\Sigma}\left(x_{0}\right), \varlimsup_{\lim _{T \rightarrow+\infty}}\left(A^{T}\left(\underset{z}{z}, f_{2}\left(x_{T}\right)\right)-A^{T}(\underset{z}{z}, 0)\right) \leq 0$.

Remark that Convention I circumvents the indeterminacy that could arise when $A^{T}(z, 0)$ and $A^{T}\left(z, f_{i}\left(x_{T}\right)\right), i \in\{1,2\}$, are simultaneously infinite.

Property T1 says that the payoff from $t=0$ to time $T$ is greater, asymptotically, if $f_{1}$ is used instead of 0 to evaluate payoff at time T. Similarly, Property T2 says that the payoff from $t=0$ to time $T$ is greater, asymptotically, if 0 is used instead of $f_{2}$ to evaluate payoff at time $T$.

Transversality allows to make clear the relationship between optimization problems $(\bar{P}),(\underline{P})$ and $(P)$ (for the proof, see the appendix).

Proposition 3.2. Assume Increasing Monotonicity Assumption (IM), let a pair $(\underline{v}, \bar{v})$ satisfy the Transversality Assumption and $U$ be a A-recursive payoff. If, for every $x_{0} \in X$ and every $z \in \Sigma\left(x_{0}\right), U(z) \in\left[\underline{v}\left(x_{0}\right), \bar{v}\left(x_{0}\right)\right]$, then for every $\underset{\sim}{z} \in \Sigma\left(x_{0}\right), U(\underset{\sim}{z})=\lim _{n \rightarrow+\infty} A^{n}(\underset{\sim}{z}, 0)$. In particular,

$$
\bar{v}^{*}\left(x_{0}\right)=\underline{v}^{*}\left(x_{0}\right)=\sup _{z \in \Sigma\left(x_{0}\right)} U(z) .
$$

\section{DYNAMIC PROGRAMMING}

Recall that $V=\mathscr{F}(X,[-\infty,+\infty])$ denotes the set of functions from $X$ to $[-\infty,+\infty]$

Definition 4.1. The Bellman operator $B: V \rightarrow V$ is defined, for every $v \in V$ and every $x \in X$, by

$$
B v(x):=B(v)(x)=\sup _{y \in \Gamma(x)}\left\{\sup _{z \in \Omega(x, y)} A(z, v(y))\right\}
$$

In this section we prove that under some weak uniform continuity on the aggregator (called Uniform continuity in $v$ ), the optimal values of the problems $(\underline{P})$ and $(\bar{P})$ are fixed points of the Bellman operator (Theorem 4.1).

We also get, under a weaker continuity assumption and transversality conditions, existence, uniqueness and computation of the solution to the Bellman equation (Theorem 4.2). This allows for encompassing approaches which were, up to now, considered as being distinct (biconvergence vs lipschitz-like approaches). 


\subsection{Existence of A solution to the Bellman Equa- TION UNDER UNIFORM CONTINUITY PROPERTY IN $v$}

In this section, we introduce some regularity assumption on the aggregator, called uniform continuity in $v$. We will prove that this assumption, together with Boundary condition and Increasing Monotonicity Assumption, implies that the value functions $\underline{v}^{*}$ and $\bar{v}^{*}$ are fixed points of the Bellman operator. In addition, if $Z$ is assumed to be a metric space and $\Sigma\left(x_{0}\right)$ is assumed to be compact for the product topology, then the same result can be obtained under a weaker assumption that we call compact uniform continuity in $v$.

\subsubsection{UNIFORM CONTINUITY IN $v$}

Assumption UC. [Uniform continuity in $v$ ] The mapping $A$ is said to be uniformly continuous in $v$ if for every $\varepsilon>0$, there exists $\eta>0$ such that for every $(z, v) \in Z \times]-\infty,+\infty\left[\right.$, for every $\eta^{\prime} \in[-\eta, \eta],\left|A\left(z, v+\eta^{\prime}\right)-A(z, v)\right| \leq \varepsilon$

If we assume that $A$ is uniformly continuous in $v$, then from Convention I, we get that if $A(z, v)=-\infty$ for some $(z, v) \in Z \times]-\infty,+\infty$ [, then $A\left(z, v+\eta^{\prime}\right)=A(z, v)=-\infty$ for every $\eta^{\prime} \in[-\eta, \eta]$. In particular, this implies that for such fixed $z$, we have $A\left(z, v^{\prime}\right)=-\infty$ for every $\left.v^{\prime} \in\right]-\infty,+\infty[$.

A similar result can be obtained for $+\infty$.

Lemma 4.1. The mapping $A$ is uniformly continuous in $v$ if and only if there exists a function $\delta: \mathbf{R} \rightarrow \mathbf{R} \cup\{+\infty\}$ that converges to 0 at 0 and such that, for every $\left(z, v, v^{\prime}\right) \in Z \times \mathbf{R} \times \mathbf{R},\left|A(z, v)-A\left(z, v^{\prime}\right)\right| \leq \delta\left(v-v^{\prime}\right)$.

Note that for every $\lambda>0, \delta(v)=\lambda|v|$ allows to recover the $\lambda$-Lipschitz case.

Example 4.1. (A class of bounded and possibly discontinuous aggregators) Assume $Z$ is a compact metric space, and let $A: Z \times[-\infty,+\infty] \rightarrow \mathbf{R}$ be such that $\left(z, v, v^{\prime}\right) \in Z \times[-\infty,+\infty]^{2} \rightarrow A(z, v)-A\left(z, v^{\prime}\right)$ is continuous ${ }^{5}$ (under

\footnotetext{
${ }^{5}$ The space $[-\infty,+\infty]$ is endowed with its standard compactification-topology: a neighbourhood of $x \in \mathbf{R}$ is standard, a neighbourhood of $-\infty$ contains $[-\infty, y$ [ for some $y \in \mathbf{R}$, and a neighbourhood of $+\infty$ contains $] y,+\infty]$ for some $y \in \mathbf{R}$.
} 
Convention I). Then $A$ is uniformly continuous in $v$. Indeed, there would otherwise exist $\varepsilon>0$ and a sequence $\left(z^{n}, v^{n}, w^{n}\right) \in Z \times[-\infty,+\infty] \times[-\infty,+\infty]$ with $\left|A\left(z^{n}, v^{n}\right)-A\left(z^{n}, w^{n}\right)\right| \geq \varepsilon$ and such that $\left|v^{n}-w^{n}\right|$ converges to 0 when $n$ tends to $+\infty$. By compactness, it can be assumed that $\left(z^{n}, v^{n}, w^{n}\right)$ converges to some $(z, v, v) \in Z \times[-\infty,+\infty] \times[-\infty,+\infty]$, and by continuity, $|A(z, v)-A(z, v)|=0 \geq \varepsilon$, a contradiction.

Examples of such aggregators are $A(z, v)=u(z)+f(v)$, for $u$ any function from $Z$ to $\mathbf{R}$ and $f:[-\infty,+\infty] \rightarrow \mathbf{R}$ continuous, non-decreasing with bounded values. This allows $A$ to present some discontinuities.

Example 4.2. (A class of additively separable aggregators satisfying a uniform continuity condition) Let $Z$ be any set and $A(z, v)=u(z)+f(v)$ for some function $u$ from $Z$ to $[-\infty,+\infty]$, and $f: \mathbf{R} \rightarrow \mathbf{R}$ a non-decreasing uniformly continuous function. Extend $A$ on $Z \times[-\infty,+\infty]$ by $A(z,-\infty)=$ $\lim _{v \rightarrow-\infty} A(z, v)$ and $A(z,+\infty)=\lim _{v \rightarrow+\infty} A(z, v)$. Such an $A$ is uniformly continuous in $v$. The function $f$ above needs not to be Lipschitz: consider for example $A(z, v)=-z^{2}+\sqrt{v}$ if $v \geq 0$ and $A(z, v)=-z^{2}$ otherwise.

Example 4.3. (Aggregators with a functional discount rate: see Jaśkiewicz, Matkowski and Nowak [5])

Consider a continuous aggregator $A$ satisfying

$$
\forall\left(z, v^{\prime}, v^{\prime \prime}\right) \in Z \times \mathbf{R}^{2},\left|A\left(z, v^{\prime}\right)-A\left(z, v^{\prime \prime}\right)\right| \leq \delta\left(\left|v^{\prime}-v^{\prime \prime}\right|\right)
$$

with $\delta$ continuous, increasing and such that for every $v \in \mathbf{R}, \lim _{n \rightarrow+\infty} \delta^{n}(|v|)=$ 0. This implies that $A$ is uniformly continuous in $v$, and that any pair of bounded functions satisfies transversality conditions.

Jaśkiewicz Matkowski and Nowak [5] treat the case of aggregators of the form $A(z, v)=u(z)+f(v)$, such that the following assumption is true: (A1) there exists a continuous increasing function $\delta:[0,+\infty[\rightarrow[0,+\infty[$ that satisfies, for every $v>0, \delta(v)<v$, and for every $\left(v, v^{\prime}\right) \in \mathbf{R}^{2},\left|f(v)-f\left(v^{\prime}\right)\right| \leq \delta\left(\left|v-v^{\prime}\right|\right)$. Condition (A1), or also the assumption that $\delta$ is strictly contracting, ensure that $\lim _{n \rightarrow+\infty} \delta^{n}(|v|)=0$. 


\subsubsection{Compact Uniform CONTINUity IN $v$}

As a matter of fact, when $Z$ is a metric space, and $\Sigma\left(x_{0}\right)$ is compact for every $x_{0} \in X$, uniform continuity in $v$ can be weakened into the following notion, called compact uniform continuity in $v$, which encompasses several interesting classes of aggregators.

Assumption CUC. [compact uniform continuity in $v$ ] Assume $Z$ is a metric space. The mapping $A$ is said to be compactly uniformly continuous in $v$ if for every compact subset $K \subset Z$, for every $\varepsilon>0$, there exists $\eta>0$ such that for every $(z, v) \in K \times \mathbf{R}$, for every $\eta^{\prime} \in[-\eta, \eta],\left|A\left(z, v+\eta^{\prime}\right)-A(z, v)\right| \leq \varepsilon$.

The following lipschitz-like property, generally easy to check in practice, implies compact uniform continuity, if one additionally assumes continuity of $A$ (for the proof, see the appendix):

Proposition 4.1. Assume $Z$ is a metric space, $A$ is continuous, and that there exists $\Lambda \geq 0$ and $f: Z \rightarrow\left[0,+\infty\left[\right.\right.$ bounded $^{6}$ on every compact of $Z$, such that for every $\left(v, v^{\prime}\right) \in \mathbf{R}^{2}$ with $|v| \geq \Lambda$ and $\left|v^{\prime}\right| \geq \Lambda$, and for every $x \in Z$, we have

$$
\left|A(z, v)-A\left(z, v^{\prime}\right)\right| \leq f(z)\left|v-v^{\prime}\right|
$$

Then $A$ is compactly uniformly continuous in $v$.

The condition above is similar to some Lipschitz condition in $v$, where the Lipschitz constant is replaced by a function of $z$ that is assumed to be bounded when $z$ varies in a compact subset of $Z$.

In practice, a possible way to obtain the criterium of this proposition is to use the mean value theorem: see Example 4.5 for an illustration.

Example 4.4. (Blackwell and Thompson aggretators, see Marinacci and Montrucchio [10]) In their paper, the authors consider two particular subclasses of aggregators: (1) continuous Blackwell aggregators and (2) continuous Thompson aggregators.

Being Lipschitz continuous in the second variable, Blackwell aggregators are straightforwardly uniformly continuous in v.

\footnotetext{
${ }^{6}$ this boundedness condition is for example true when $f$ is continuous.
} 
By definition, a Thompson ${ }^{7}$ aggregator $A:[0,+\infty[\times[0,+\infty[\rightarrow \mathbf{R}$ satisfies the following properties (see [10], page 1783):

1. $A$ is nonnegative and monotone.

2. Equation $A(z, v)=v$ has a unique ${ }^{8}$ solution $v=a_{z}$ for each $z>0$.

3. $A$ is concave at 0 for each $z \geq 0$, that is

$$
\forall \alpha \in[0,1], A(z, \alpha v) \geq \alpha A(z, v)+(1-\alpha) A(z, 0)
$$

The following proposition, proved in the appendix, says that compact uniform continuity assumption encompasses the class of Thompson aggregators.

Proposition 4.2. If $A$ is a continuous Thompson aggregator, then it is compactly uniformly continuous in $v$.

Example 4.5. Consider the family of aggregators defined on $\left[0,+\infty\left[^{2}\right.\right.$

$$
A(z, v)=\left(z^{\eta}+\beta v^{\sigma}\right)^{\frac{1}{\rho}}
$$

In [10], it is proved that (1) $A$ is a Thompson aggregator if $\sigma \leq 1$ and either $\sigma<\rho$ or $[\sigma=\rho$ and $\beta<1]$ (2) $A$ is a Blackwell aggregator if $\beta<1 \leq \sigma=\rho$. For such values, the previous criterium can apply. As a matter of fact, compact uniform continuity assumption encompasses additional cases: as an illustration, consider $A(z, v)=\left(z+v^{2}\right)^{\frac{1}{3}}$. It is not a Thompson aggregator, nor a Blackwell aggregator, but it is compactly uniformly continuous in $v$. Indeed, the mean value theorem applied to $v \rightarrow A(z, v)=\left(z+v^{2}\right)^{\frac{1}{3}}$ gives $\forall\left(v, v^{\prime}\right) \in\left[0,+\infty\left[^{2}, v<v^{\prime},\left|A(z, v)-A\left(z, v^{\prime}\right)\right| \leq \max _{v^{\prime \prime} \in\left[v, v^{\prime}\right]} \frac{2 v^{\prime \prime}}{3}\left(z+v^{\prime \prime 2}\right)^{-\frac{2}{3}}\left|v-v^{\prime}\right|\right.\right.$.

that is

$\forall\left(v, v^{\prime}\right) \in\left[0,+\infty\left[^{2}, v<v^{\prime},\left|A(z, v)-A\left(z, v^{\prime}\right)\right| \leq \max _{v^{\prime \prime} \in\left[v, v^{\prime}\right]} \frac{2}{3} v^{\prime \prime-\frac{1}{3}}\left(\frac{z}{v^{\prime \prime 2}}+1\right)^{-\frac{2}{3}}\left|v-v^{\prime}\right|\right.\right.$,

thus taking $\Lambda=1$, for $v \geq \Lambda$ and $v^{\prime} \geq \Lambda$, we obtain

$$
\left|A(z, v)-A\left(z, v^{\prime}\right)\right| \leq \frac{2}{3}\left|v-v^{\prime}\right|
$$

which proves (by Proposition 4.1) compact uniform continuity in $v$.

\footnotetext{
${ }^{7}$ Note that a Thompson aggregator $A$ is assumed to be defined on $Z \times[0,+\infty[$, and not on $Z \times \mathbf{R}$ as in our paper. It is easy to extend the aggregators to the case of this paper, so that we can apply the main results of our paper to this class of aggregators.

${ }^{8}$ In the definition given by Marinacci et al. [10], it is only required that there exists at least such an $a_{z}$, but the authors prove uniqueness in Lemma 1 (page 1798).
} 


\subsubsection{The EXISTENCE RESUlT}

The existence result is detailed in the following statement (for the proof, see the appendix):

Theorem 4.1. Assume the aggregator satisfies Increasing Monotonicity and Boundary assumptions. If one of the two additional assumptions i) or ii) are satisfied, then $B \underline{v}^{*}=\underline{v}^{*}$ and $B \bar{v}^{*}=\bar{v}^{*}$.

i) A is uniformly continuous in $v$.

ii) $Z$ is a metric space, for every $x_{0} \in X, \Sigma\left(x_{0}\right)$ is compact, and $A$ is compactly uniformly continuous in $v$.

The following example proves that this result can be false if the aggregator is neither uniformly continuous in $v$ nor compactly uniformly continuous in $v$.

Example 4.6. Consider the aggregator defined in the proof of Proposition 3.1 , by $Z=\mathbf{R}, A(z, v)=v / 2+1 / 2-z^{2}$ if $v<1$ and $A(z, v)=v+1-z^{2}$ otherwise. But for any $\eta>0,|A(z, 1-\eta)-A(z, 1)| \geq 1 / 2$, hence $A$ is neither uniformly continuous in $v$ nor compactly uniformly continuous in $v$. Assume correspondences $\Gamma$ and $\Omega$ are such that $\Sigma\left(x_{0}\right)$ contains the null sequence for every $x_{0} \in X$, the value function $\bar{v}^{*}$ is obtained for $\mathbf{0}$ and equals 1 . But $B(1)\left(x_{0}\right)=2$, thus the value function $\bar{v}^{*}$ is not a fixed-point of the Bellman operator.

The assumptions of this theorem cover most of the aggregators found in the literature (see Examples 4.2 and 4.1).

\subsection{Existence, Uniqueness AND COMPUtation OF A SOlU- TION TO THE BELLMAN EQUATION UNDER WEAK CON- TINUITY}

The aim of this section is to refine Theorem 4.1 in order to get some uniqueness result, provide an algorithm to compute the solution, and encompass the aggregators defined from the biconvergent recursive payoff functions as introduced by Streufert [19], [20]. 
This is done through transversality and a weak continuity assumption (which is a refinement of the uniform continuity in $v$ assumption).

\subsubsection{WEAK CONTINUITY}

Definition 4.2. Consider a function $\bar{v}$ from $X$ to $[-\infty,+\infty]$. Define the function $f:[0,1]^{\mathbf{N}} \rightarrow[-\infty,+\infty]$ by

$$
\begin{aligned}
f(\varepsilon)=\sup _{(z, x) \in \widetilde{\Sigma}\left(x_{0}\right)} & \inf _{n \in \mathbf{N}^{*}} A\left(z_{0}, A\left(z_{1}, A\left(z_{2}, \ldots\right.\right.\right. \\
& \left.\left.\left.\left.\ldots, A\left(z_{n-1}, \bar{v}\left(x_{n}\right)\right)+\varepsilon_{n-1}\right)+\varepsilon_{n-2}+\cdots\right)+\varepsilon_{2}\right)+\varepsilon_{1}\right),
\end{aligned}
$$

where $\varepsilon=\left(\varepsilon_{i}\right)_{i \in \mathbf{N}^{*}}$. The aggregator $A$ is said to be weakly continuous at $\bar{v}$ if the function $f$ is upper semicontinuous at $0 .{ }^{9}$

Remark 4.1. 1) The price to pay for the generality of this assumption is its complexity: it is less testable than Uniform continuity. Uniform continuity in $v$ implies weak continuity at $\bar{v}: X \rightarrow[-\infty,+\infty]$, whatever $\bar{v}$ (see the next proposition).

2) Weak continuity implies that $f$ is continuous at 0 , lower semicontinuity at 0 being a consequence of the Increasing Monotonicity Assumption.

3) The interpretation of the weak continuity assumption is the following. Assume that your terminal payoff at $t=n$ is valued with $\bar{v}$ and imagine a prudent entity that tries to maximize the worst possible payoff from 0 to $n$, $n$ spanning $\mathbf{N}$. Then, roughly, weak continuity says that the optimal value of this prudent entity should vary continuously at 0 with respect to small additive perturbations of payoffs through time.

The following propositions (for proofs, see the appendix) prove that weak continuity refines Uniform continuity in $v$, as well as it refines Compact Uniform continuity in $v$ when feasibility sets are compact.

Proposition 4.3. If an aggregator $A$ is uniformly continuous in $v$, then it is weakly continuous at $\bar{v}$ for every $\bar{v}: X \rightarrow[-\infty,+\infty]$.

\footnotetext{
${ }^{9}$ For some well-suited topology on $[0,1]^{\mathbf{N}}$ such that every neighborhood of 0 intersect $[0,1]^{\mathbf{N}}$. This technical requirement would then provide a meaning to $\lim _{\varepsilon \rightarrow 0, \varepsilon \neq 0} f(\varepsilon)$, i.e., a limit whose existence is required from the proof of the main current Theorem $4.2, \mathbf{R}$ being currently endowed with the standard metric.
} 
Proposition 4.4. If an aggregator $A$ is compactly uniformly continuous in $v$, and if $\Sigma\left(x_{0}\right)$ is compact (for the product topology) for every $x_{0} \in X$, then it is weakly continuous at $\bar{v}$ for every $\bar{v}: X \rightarrow[-\infty,+\infty]$.

\subsubsection{Existence, Uniqueness And COMPUTATION OF THE SOLUTiON TO THE BELLMAN EQUATION}

The following theorem (for the proof, see the appendix) shows that transversality and weak continuity allow to get existence, uniqueness and computation of the fixed point of the Bellman operator.

Theorem 4.2. Assume Increasing Monotonicity Assumption. For every $(\underline{v}, \bar{v}) \in V^{2}$ satisfying Transversality, with $\underline{v}\left(x_{0}\right) \leq \bar{v}\left(x_{0}\right)<+\infty$ for every $x_{0} \in X$, and such that $A$ is weakly continuous ${ }^{10}$ at $\bar{v}$, we have:

1. Assume there exists $v \in[\underline{v}, \bar{v}]$ such that $B v=v$. Then

a. [Uniqueness] $v=\bar{v}^{*}=\underline{v}^{*}$.

b. [Computation] For every $f \leq v$ satisfying Transversality Assumption (T1), one has $\overline{\lim }_{n \rightarrow+\infty} B^{n} f=v$.

2. [Existence] If $\underline{v}$ and $\bar{v}$ also satisfy $B \underline{v} \geq \underline{v}$ and $B \bar{v} \leq \bar{v}$, then there exists a (unique) fixed-point of $B$ in $[\underline{v}, \bar{v}]$.

Thus, under the above assumptions, Bellman equation provides the (unique) value function of $\bar{P}$ and $\underline{P}$.

\subsubsection{ON THEOREMS 4.1 AND 4.2}

Theorem 4.2 and Theorem 4.1 both give conditions to get the existence of a solution to the Bellman equation. These conditions are however not comparable in general: on one hand, weak continuity is weaker than uniform continuity in $v$, but on the other hand, Theorem 4.2 requires to be able to identify an interval $[\underline{v}, \bar{v}]$ such that $B([\underline{v}, \bar{v}]) \subset[\underline{v}, \bar{v}]$. Thanks to Theorem 4.1, we now introduce a method that could provide such an interval.

Definition 4.3 (Aggregators dominated by well behaved aggregators). A model $(A, \Gamma, \Omega)$ is said to be dominated by another model $\left(A^{\prime}, \Gamma^{\prime}, \Omega^{\prime}\right)$ (written $\left.(A, \Gamma, \Omega) \lesssim\left(A^{\prime}, \Gamma^{\prime}, \Omega^{\prime}\right)\right)$ if $A \leq A^{\prime}, \Gamma \subset \Gamma^{\prime}$ and $\Omega \subset \Omega^{\prime}$.

\footnotetext{
${ }^{10}$ This is true, in particular, when $A$ is uniformly continuous in $v$
} 
Proposition 4.5. For an aggregator $A$, denote by $v_{A}^{*}$ the upper value associated to A. Assume $(\underline{A}, \underline{\Gamma}, \underline{\Omega}) \lesssim(A, \Gamma, \Omega) \lesssim(\bar{A}, \bar{\Gamma}, \bar{\Omega})$. If $\underline{A}, \bar{A}$ and $A$ are uniformly continuous in $v$, satisfy Increasing Monotonicity and Boundary assumption, if $\left(v_{\underline{A}}^{*}, v_{\bar{A}}^{*}\right) \in V^{2}$ satisfies Transversality, and if $v_{\bar{A}}^{*}\left(x_{0}\right)<+\infty$ for every $x_{0} \in X$, then $v_{A}^{*}$ is the (unique) fixed-point in $\left[v_{\underline{A}}^{*}, v_{A}^{*}\right]$ of the Bellman operator associated to $A$.

Proof. Let $B^{A}, B^{\bar{A}}$ and $B^{\underline{A}}$ be the Bellman operators associated to the aggregators $A, \bar{A}$ and $\underline{A}$. Clearly $v_{\bar{A}}^{*} \geq v_{\underline{A}}^{*}$. Moreover, $B^{A}\left(v_{\underline{A}}^{*}\right) \geq B^{\underline{A}}\left(v_{\underline{A}}^{*}\right)=v_{\underline{A}}^{*}$ (from Theorem 4.1, $v_{\underline{A}}^{*}$ is indeed a fixed point of the operator $B^{\underline{A}}$ ), and similarly, $B^{A}\left(v^{*}\right) \leq v_{A}^{*}$. Thus, from Theorem 4.2 (the other assumptions being easily checked), $v_{A}^{*}$ is the only fixed-point of $B^{A}$ in $\left[v_{\underline{A}}^{*}, v_{\bar{A}}^{*}\right]$.

QED

\subsubsection{AdDitive AgGregators And BICONVERGEnt AGgREgATors}

As an illustration, consider now the Additive Aggregator case:

Example 4.7. (Additive Aggregator: Kamihigashi [6]). Theorem 4.2 allows for recovering one part ${ }^{11}$ of the results of Kamihigashi [6]. Following the notations of Kamihigashi, let $X$ be a set, $\Gamma$ be a nonempty-valued correspondence from $X$ to $X, D=\{(x, y): y \in \Gamma(x)\}$ the graph of $\Gamma$, $u: D \rightarrow[-\infty,+\infty[$ and $\beta \geq 0$. We now define $Z=X \times X, \Omega(z)=\{z\}$, $A(z, v)=u(z)+\beta v$ for every $z \in Z$ and $v \in]-\infty,+\infty[$. The aggregator $A$ can be extended on $Z \times[-\infty,+\infty]$ by letting $A(z,-\infty)=\lim _{v \rightarrow-\infty} A(z, v)$ and $A(z,+\infty)=\lim _{v \rightarrow+\infty} A(z, v)$.

Consider the two following sets

$$
\begin{aligned}
& \Pi\left(x_{0}\right)=\left\{\left(x_{t}\right)_{t \in \mathbf{N}^{*}} \in X^{\mathbf{N}}: \forall t \in \mathbf{N}, x_{t+1} \in \Gamma\left(x_{t}\right)\right\} \\
& \Pi^{0}\left(x_{0}\right)=\left\{\left(x_{t}\right)_{t \in \mathbf{N}^{*}} \in \Pi\left(x_{0}\right): \varlimsup_{\lim _{T \rightarrow \infty}} \sum_{t=0}^{T} \beta^{t} u\left(x_{t}, x_{t+1}\right)>-\infty\right\},
\end{aligned}
$$

Suppose, as in T. Kamihigashi [6], Theorem 2.1., that there exist $(\underline{v}, \bar{v}) \in V^{2}$ such that $\underline{v} \leq \bar{v}$, with $B \underline{v} \geq \underline{v}, B \bar{v} \leq \bar{v}$ and

$$
\forall\left(x_{t}\right)_{t \in \mathbf{N}} \in \Pi^{0}\left(x_{0}\right), \underline{\lim }_{t \rightarrow+\infty} \beta^{t} \underline{v}\left(x_{t}\right) \geq 0,
$$

\footnotetext{
${ }^{11}$ Actually, Kamihigashi considers a family of optimization problems, parametrized by $L \in\{\underline{\lim }, \varlimsup \lim \}$, and in this subsection, we only derive the supremum limit case.
} 


$$
\forall\left(x_{t}\right)_{t \in \mathbf{N}} \in \Pi\left(x_{0}\right), \varlimsup_{t \rightarrow+\infty} \beta^{t} \bar{v}\left(x_{t}\right) \leq 0 .
$$

The Increasing Monotonicity Assumption is satisfied, $A$ is uniformly continuous in $v$. Moreover, Equations 4.1 and 4.2 imply that the pair $(\underline{v}, \bar{v})$ satisfies the Transversality assumption, since one has for every $(z, x) \in \widetilde{\Sigma}^{0}\left(x_{0}\right)$ :

$$
A^{T}\left(z, \underline{v}\left(x_{T}\right)\right)-A^{T}(z, 0)=\beta^{T} \underline{v}\left(x_{T}\right)
$$

and similarly for $\bar{v}$. By Theorem 4.2, $\bar{v}^{*}$ is the unique fixed-point of $B$. Moreover, taking $f=\underline{v}$ in Theorem 4.2, the sequence $\left(B^{n} \underline{v}\right)_{n}$ converges to $\bar{v}^{*}$ for the pointwise convergence.

Example 4.8. (Biconvergence aggregators, Streufert's Section 3.3 Theorem D [19], and Streufert's Section 3.4 Theorem B [20])

In $[19,20]$, Streufert proves that there exists a unique solution to Bellman equation under a new topological notion he calls Biconvergence. We now explain how deriving a part of his results from Theorem 4.2. Assume now that $Z=X$ and $\Omega(x, y)=\{y\}$. A model is then a pair $(A, \Gamma)$, the aggregator $A$ maps $X \times[-\infty,+\infty]$ to $[-\infty,+\infty]$, and $\Sigma\left(x_{0}\right)=\left\{\left(x_{1}, x_{2}, \ldots\right) \in X^{\mathbf{N}}: \forall t \in\right.$ $\left.\mathbf{N}, x_{t+1} \in \Gamma\left(x_{t}\right)\right\}$.

The Bellman operator $B: \mathscr{F}(X,[-\infty,+\infty]) \rightarrow \mathscr{F}(X,[-\infty,+\infty])$ is then defined, for every $v \in \mathscr{F}(X,[-\infty,+\infty])$ and every $x \in X$, by:

$$
B v(x)=\sup _{y \in \Gamma(x)} A(y, v(y)) .
$$

Definition 4.4. Let $U$ be a $A$-recursive payoff. The function $U$ is said to be upper convergent over $\prod_{t=1}^{+\infty} \Gamma^{t}\left(x_{0}\right)$ if

$$
\forall x \in \prod_{t=1}^{+\infty} \Gamma^{t}\left(x_{0}\right), \lim _{t \rightarrow+\infty}\left(\sup U\left(x_{1}, \ldots, x_{t}, \prod_{s=t+1}^{+\infty} \Gamma^{s}\left(x_{0}\right)\right)\right)=U(\underset{\sim}{x})
$$

The function $U$ is said to be lower convergent over $\prod_{t=1}^{+\infty} \Gamma^{t}\left(x_{0}\right)$ if

$$
\forall x \in \prod_{t=1}^{+\infty} \Gamma^{t}\left(x_{0}\right), \lim _{t \rightarrow+\infty}\left(\inf U\left(x_{1}, . ., x_{t}, \prod_{s=t+1}^{+\infty} \Gamma^{s}\left(x_{0}\right)\right)=U(\underset{\sim}{x})\right.
$$

The function $U$ is biconvergent over $\prod_{t=1}^{+\infty} \Gamma^{t}\left(x_{0}\right)$ if it is both upper and lower convergent over $\prod_{t=1}^{+\infty} \Gamma^{t}\left(x_{0}\right)$.

Another equivalent definition is (see appendix for the equivalence): 
Definition 4.5. The function $U$ is biconvergent over $\prod_{t=1}^{+\infty} \Gamma^{t}\left(x_{0}\right)$ if and only if for every $\underset{\sim}{x} \in \prod_{t=1}^{+\infty} \Gamma^{t}\left(x_{0}\right)$, for every $\varepsilon>0$, there exists $N \in \mathbf{N}$ such that for every $\left(x_{N+1}^{\prime}, x_{N+2}^{\prime}, \ldots\right) \in \prod_{t=N+1}^{+\infty} \Gamma^{t}\left(x_{0}\right)$, one has:

$$
\left|U\left(x_{1}, x_{2}, \ldots, x_{N}, x_{N+1}, x_{N+2}, \ldots\right)-U\left(x_{1}, x_{2}, \ldots, x_{N}, x_{N+1}^{\prime}, x_{N+2}^{\prime}, \ldots\right)\right| \leq \varepsilon .
$$

Otherwise stated, $U$ is biconvergent over $\prod_{t=1}^{+\infty} \Gamma^{t}\left(x_{0}\right)$ if for every $\varepsilon>0$, there exists some time $N$ such that the utility $U$ does not move of more that $\varepsilon$ if the (feasible) actions are modified after time $N$.

The following result, obtained by Streufert [19], emerges as a Corollary of Theorem 4.2 (for a proof, see the appendix):

Corollary 4.1. Consider a model $(A, \Gamma)$, where $A: X \times[-\infty,+\infty] \rightarrow$ $[-\infty,+\infty]$ is an upper semicontinuous aggregator, and $\Gamma$ is an upper semicontinuous multivalued function from $X$ to $X$, with compact values. Let $U$ be a A-recursive payoff. Assume Increasing Monotonicity and Null Assumption. Last, assume that $\max U\left(\prod_{t=1}^{+\infty} \Gamma^{t}\left(x_{0}\right)\right)$ exists in $\mathbf{R}$, and that $U$ is biconvergent over $\prod_{t=1}^{+\infty} \Gamma^{t}\left(x_{0}\right)$, for some $x_{0} \in X$. Then $\bar{v}^{*}: X \rightarrow \mathbf{R}$ defined by $\bar{v}^{*}\left(x_{0}\right)=\sup _{z \in \Sigma\left(x_{0}\right)} U(z)$ is the unique solution to the Bellman equation.

\section{A. Proof of Proposition 2.1.}

Let $Z=\mathbf{R}$. Define $A$ on $\mathbf{R} \times\left[-\infty,+\infty\left[\right.\right.$ by $A(z, v)=\frac{v}{2}+\frac{1}{2}+z$ if $\left.v \in\right]-\infty, 1[$, $A(z, v)=v+1+z$ if $v \in[1,+\infty[$ and $A(z,-\infty)=-\infty$.

For every $z_{0} \leq 0$, $A\left(z_{0}, A\left(z_{0}, 0\right)\right)=A\left(z_{0}, \frac{1}{2}+z_{0}\right)=\frac{1}{2}\left(z_{0}+\frac{1}{2}\right)+\frac{1}{2}+z_{0}=\frac{1}{2}+\frac{1}{4}+z_{0}\left(1+\frac{1}{2}\right)$,

and iterating, we get:

$$
A^{n}\left(z_{0}, \ldots, z_{0}, 0\right)=\sum_{k=1}^{n} \frac{1}{2^{k}}+z_{0} \sum_{k=0}^{n-1} \frac{1}{2^{k}} .
$$

In particular,

$$
\lim _{n \rightarrow+\infty} A^{n}(\mathbf{0}, 0)=1
$$

with $\mathbf{0}$ the null sequence, and

$$
\lim _{n \rightarrow+\infty} A^{n}(-\mathbf{1}, 0)=-1
$$


with $\mathbf{- 1}$ the constant sequence whose terms are all -1 .

And one also has

$$
\lim _{n \rightarrow+\infty} A^{n}(\mathbf{1}, 0)=\lim _{n \rightarrow+\infty}\left(\frac{1}{2}+2 n+1\right)=+\infty
$$

with 1 the constant sequence whose terms are all +1 .

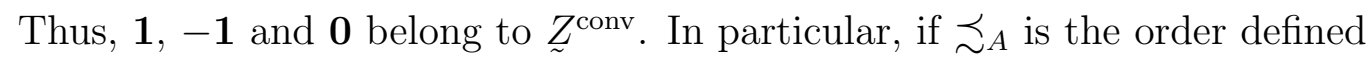
by $A$ on $\underset{\sim}{Z}$ conv , and $\precsim_{A}$ is the strict order associated ${ }^{12}$ to $\precsim_{A}$, it is obtained that:

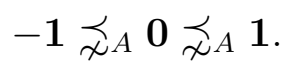

We now prove by contradiction the Proposition for $A$ defined above: assume there exists a $A$-recursive function $U$ which induces the same order. One should have

$$
U(-\mathbf{1})<U(\mathbf{0})<U(\mathbf{1})
$$

But since $U$ is $A$-recursive,

$$
A(0, U(\mathbf{0}))=U(\mathbf{0})
$$

First assume $U(\mathbf{0})$ finite. Then if $U(\mathbf{0}) \geq 1$, the last equality can be written

$$
U(\mathbf{0})+1=U(\mathbf{0})
$$

a contradiction. Otherwise, if $U(\mathbf{0})<1$, one gets

$$
\frac{U(\mathbf{0})}{2}+\frac{1}{2}=U(\mathbf{0})
$$

and $U(\mathbf{0})=1$, a contradiction. Consequently, $U(\mathbf{0})=+\infty$ or $U(\mathbf{0})=-\infty$, which contradicts $U(-\mathbf{1})<U(\mathbf{0})<U(+\mathbf{1})$. This proves that we do not have $\mathbf{- 1} \precsim_{U} \mathbf{0} \precsim_{U} \mathbf{1}$, i.e. $\precsim_{U}$ and $\precsim_{A}$ are different.

\section{B. Proof of Proposition 3.1.}

1. Let $Z=\mathbf{R}$ and assume that there is no feasibility constraints. Define $A$ by $A(z, v)=\frac{v}{2}+\frac{1}{2}-(z)^{2}$ if $\left.v \in\right]-\infty, 1\left[, A(z, v)=v+1-(z)^{2}\right.$ if $v \in[1,+\infty[$,

\footnotetext{
${ }^{12}$ that is, $x \precsim A y$ if $x \precsim A y$ is true and $y \precsim A x$ is false.
} 
$A(z,+\infty)=+\infty$ and $A(z,-\infty)=-\infty$. Then

$$
A^{n}(z, 0)=\sum_{k=1}^{n} \frac{1}{2^{k}}-\sum_{k=0}^{n-1} z_{k}^{2} \frac{1}{2^{k}} .
$$

The optimal value of $(\bar{P})$ is 1 , and the maximum is reached only at $\mathbf{0}$, the null sequence, i.e., the set of solutions is $\operatorname{Sol}(\bar{P})=\{\mathbf{0}\}$.

Let us show that $\operatorname{Sol}(P) \neq\{\mathbf{0}\}$.

If $\operatorname{Sol}(P)=\emptyset$, then clearly $\operatorname{Sol}(P) \neq\{\mathbf{0}\}$. If $\operatorname{Sol}(P) \neq \emptyset$, the maximum of $U$ is reached at some $z \in \Sigma\left(x_{0}\right)$. Let us then prove by contradiction that $U(z)$ is infinite.

Assume that $U(\underset{\approx}{z})$ is finite. Then either $U(\underset{\sim}{z})<1$ and for every small enough values of $a \in Z, U(a, z)=A(a, U(z))=\frac{1}{2} U(z)+\frac{1}{2}-a^{2}>U(z)$ a contradiction. Or for every small enough values of $a \in Z, U(z) \geq 1$, hence $U(a, z)=A(a, U(z))=U(z)+1-a^{2}>U(z)$, another contradiction. So, $U(z)$ is infinite.

If $U(z)=-\infty$ then $U$ is constantly equal to $-\infty$ on $Z^{\mathbf{N}}$, and $\operatorname{Sol}(P)=Z^{\mathbf{N}} \neq$ $\{0\}$. If $U(z)=+\infty$, then for every $a \in Z,+\infty=A(a,+\infty)=A(a, U(z))=$ $U(a, z)$, and by iteration, $U(\underset{\sim}{y})=+\infty$ for every sequence $\underset{\sim}{y}$ equal to $\underset{\sim}{z}$ except for a finite number of terms. Then $\operatorname{Sol}(P)$ is infinite and $\operatorname{Sol}(P) \neq\{\mathbf{0}\}$.

2. We now consider a general aggregator $A$ satisfying Null assumption. Thus, one has

$$
\begin{aligned}
& \sup _{z \in \Sigma\left(x_{0}\right)} \varlimsup_{\lim } \rightarrow+\infty A^{T}(z, 0)
\end{aligned}
$$

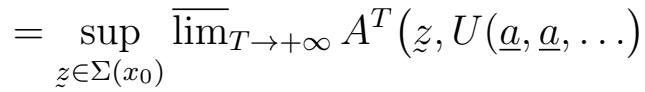

$$
\begin{aligned}
& =\sup _{z \in \Sigma\left(x_{0}\right)} \varlimsup_{T \rightarrow+\infty} U\left(z_{0}, z_{1}, \ldots, z_{T-1}, \underline{a}, \underline{a}, \ldots\right) \\
& \leq \sup _{z \in \Sigma\left(x_{0}\right)} U(z)=v^{* *}\left(x_{0}\right),
\end{aligned}
$$

since $\left(z_{0}, z_{1}, \ldots, z_{T-1}, \underline{a}, \underline{a}, \ldots\right) \in \Sigma\left(x_{0}\right)$.

To prove that such an inequality can be strict, consider an aggregator $A$ such that $A(z,+\infty)=+\infty$. For every $A$-recursive payoff $U$, one can construct a new recursive payoff $\bar{U}$ as follows: fix $z^{\prime} \in Z^{\mathbf{N}}$, and define $\bar{U}(z)=+\infty$ if $z=z^{\prime}$ but for a finite number of terms, and $\bar{U}(z)=U(z)$ otherwise. Then $\bar{U}$ is a $A$-recursive payoff, and the value of $(P)$ associated to this payoff is $+\infty$, 
which is strictly larger than the value of $(\bar{P})$ and completes the argument of the proof.

\section{Proof of Proposition 3.2.}

Let $x_{0} \in X$. We first prove that for every $z \in \Sigma\left(x_{0}\right), U(z) \geq \varlimsup_{z} \overline{\lim }_{n \rightarrow+\infty} A^{n}(z, 0)$. If $U(z)=+\infty$ or $\varlimsup_{n \rightarrow+\infty} A^{n}(z, 0)=-\infty$ then the inequality is true. Consequently, we can assume $U(z)<+\infty$ and $w(z)=\varlimsup_{\underset{\sim}{*}} \rightarrow+\infty A^{n}(z, 0)>-\infty$. Since $U$ is $A$-recursive, for every $z \in \Sigma\left(x_{0}\right)$ and $n \in \mathbf{N}$, we have $U(z)=$ $A^{n}\left(z, U\left(z_{n+1}, z_{n+2}, \ldots\right)\right)$. Since $U$ is bounded below by $\underline{v}$, and from Increasing Monotonicity Assumption, we get, choosing any $\underset{\sim}{x}$ such that $\underset{\sim}{z}, \underset{\sim}{x}) \in \widetilde{\Sigma}^{0}\left(x_{0}\right)$ (this exists because $w(z)>-\infty$ )

$$
U(z) \geq A^{n}\left(\underset{z}{z}, \underline{v}\left(x_{n}\right)\right) .
$$

Since $U(\underset{z}{z})<+\infty$, we get $A^{n}\left(\underset{z}{z}, \underline{v}\left(x_{n}\right)\right)<+\infty$ for every $n$. Let $\varepsilon>0$. From Transversality Assumption (T1), we get that for $n$ large enough,

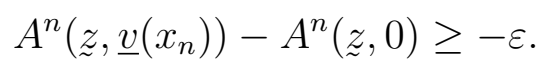

In particular, $A^{n}(z, 0)<+\infty$ for $n$ large enough.

Now, for every $n$ large enough such that $A^{n}(z, 0)>-\infty$ (thus is real),

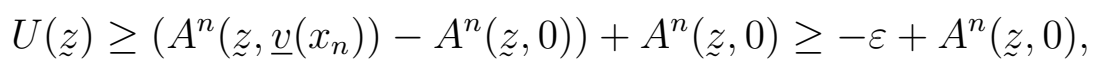

The above inequality is also true for $A^{n}(z, 0)=-\infty$, and passing to the supremum limit and taking $\varepsilon \rightarrow 0$, we get $U(z) \geq \varlimsup_{z \rightarrow+\infty} A^{n}(z, 0)$.

The same proof gives that for every $z \in \Sigma\left(x_{0}\right), U(z) \leq \underline{\lim }_{n \rightarrow+\infty} A^{n}(z, 0)$.

Thus, finally, for every $z \in \Sigma\left(x_{0}\right), U(z)=\lim _{n \rightarrow+\infty} A^{n}(z, 0)$.

\section{Proof of Lemma 4.1}

Let us show (i) $\Leftrightarrow$ (ii) where

(i) There exists $\delta: \mathbf{R} \rightarrow \mathbf{R} \cup\{+\infty\}$ that tends to 0 at 0 and such that, for every $\left(z, v, v^{\prime}\right) \in Z \times \mathbf{R} \times \mathbf{R},\left|A(z, v)-A\left(z, v^{\prime}\right)\right| \leq \delta\left(v-v^{\prime}\right)$, with convention (I), and 
(ii) $A$ is uniformly continuous in $v$.

Clearly (i) $\Rightarrow$ (ii). Let us show that (ii) $\Rightarrow$ (i). Assume $A$ is uniformly continuous in $v$ and define for every $x \in \mathbf{R}$ :

$$
\delta(x)=\sup _{(z, v) \in Z \times \mathbf{R}}\{|A(z, v+x)-A(z, v)|\}
$$

with convention (I), that is $A(z, v+x)-A(z, v)$ is taken to be equal to 0 if $A(z, v+x)=A(z, v)=+\infty$ or $A(z, v+x)=A(z, v)=-\infty$. By definition

of $\delta$, Condition (i) is true for such $\delta$, and the fact that $\delta: \mathbf{R} \rightarrow \mathbf{R} \cup\{+\infty\}$ tends to 0 at 0 comes from Condition (ii).

\section{E. Proof of Proposition 4.1.}

Assume that the criterium of Proposition 4.1 is true, but that $A$ is not compactly uniformly continuous in $v$. Thus, there is $K$ compact in $Z, \varepsilon>0$, $\left(z_{n}, v_{n}, v_{n}^{\prime}\right)$ a sequence in $K \times \mathbf{R}^{2}$ with $\left|v_{n}-v_{n^{\prime}}\right| \leq \frac{1}{n}$ and such that

$$
\left|A\left(z_{n}, v_{n}\right)-A\left(z_{n}, v_{n}^{\prime}\right)\right|>\varepsilon
$$

Since $\left|v_{n}-v_{n^{\prime}}\right| \leq \frac{1}{n}$, we have two cases:

(1) Either there is a subsequence of $\left(v_{n}, v_{n}^{\prime}\right)$ which converges. Then, up to a subsequence, we can assume that $\left(z_{n}, v_{n}, v_{n}^{\prime}\right)$ converges to some $(z, v, v) \in$ $K \times \mathbf{R}^{2}$. Passing to the limit in Equation E.1, and from the continuity of $A$, we get $\varepsilon \leq 0$, a contradiction.

(2) Or, $\left|v_{n}\right|$ and $\left|v_{n}^{\prime}\right|$ converges to $+\infty$. By assumption, for $n$ large enough, we get

$$
\varepsilon<\left|A\left(z_{n}, v_{n}\right)-A\left(z_{n}, v_{n}^{\prime}\right)\right| \leq f\left(z_{n}\right)\left|v_{n}-v_{n}^{\prime}\right| \leq f\left(z_{n}\right) \frac{1}{n}
$$

and passing to the limit, and from the boundedness of $f\left(z_{n}\right)$, we get a contradiction. 


\section{F. Proof of Proposition 4.2.}

From Property (3) of Thompson aggregators and monotonicity of $A$, we get that for every $z \geq 0$ and every $v^{\prime}>v \geq 0$,

$$
\left|\frac{A\left(z, v^{\prime}\right)-A(z, v)}{v^{\prime}-v}\right| \leq\left|\frac{A(z, v)-A(z, 0)}{v}\right|
$$

and similarly, we get, for every $z>0$ and every $v \geq a_{z}$,

$$
\left|\frac{A(z, v)-A(z, 0)}{v-0}\right| \leq\left|\frac{A\left(z, a_{z}\right)-A(z, 0)}{a_{z}-0}\right|
$$

Which finally gives for every $z>0$, every $v^{\prime}>v \geq a_{z}$,

$$
\left|A\left(z, v^{\prime}\right)-A(z, v)\right| \leq\left(v^{\prime}-v\right)\left|\frac{a_{z}-A(z, 0)}{a_{z}}\right| \leq\left(v^{\prime}-v\right)
$$

which is still true for $z=0$ by continuity of $A$.

Now, to prove that $A$ is compactly uniform continuous in $v$, assume by contradiction that there is $K$ compact in $Z, \varepsilon>0,\left(z_{n}, v_{n}, v_{n}^{\prime}\right)$ a sequence in $K \times\left[0,+\infty\left[\times\left[0,+\infty\left[\right.\right.\right.\right.$ with $\left|v_{n}-v_{n^{\prime}}\right| \leq \frac{1}{n}$ and such that

$$
\left|A\left(z_{n}, v_{n}\right)-A\left(z_{n}, v_{n}^{\prime}\right)\right|>\varepsilon \text {. }
$$

If $\left(v_{n}, v_{n}^{\prime}\right)$ has a bounded subsequence, then up to a subsequence, we can assume that $\left(z_{n}, v_{n}, v_{n}^{\prime}\right)$ converges to some $(z, v, v)$, and continuity of $A$ gives, passing to the limit in the previous inequality, $0 \geq \varepsilon$, a contradiction. Thus, $\left(v_{n}, v_{n}^{\prime}\right)$ has no bounded subsequence, and the two sequences $v_{n}$ and $v_{n}^{\prime}$ tends to $+\infty$.

Let $a_{n}$ such that $A\left(z_{n}, a_{n}\right)=a_{n}$ (such an $a_{n}$ is unique if $z_{n}>0$ but it still exists when $\left.z_{n}=0\right)$.

Since $z_{n} \in K, K$ compact, it is bounded. Let us prove that $a_{n}$ is bounded.

By contradiction, assume that $\lim _{n \rightarrow+\infty} a_{n}=+\infty$. Define $\eta>0$ and $N$ such that $n \geq N$ implies $z_{n}<z+\eta$. By assumption, since $z+\eta>0$, there exists a unique $a$ such that $A(z+\eta, a)=a$, and we have $A\left(z+\eta, a^{\prime}\right)>a^{\prime}$ for $a^{\prime}<a$ and $A\left(z+\eta, a^{\prime}\right)<a^{\prime}$ for $a^{\prime}>a$ (the function $\frac{A(z+\eta, v)}{v}$ is indeed strictly decreasing for $v \in\left[0,+\infty\left[\right.\right.$ : see Lemma 1 in [10]). Thus, if $a^{\prime}=a_{n}>a$, $A\left(z+\eta, a_{n}\right)<a_{n}$. Let $N^{\prime} \in \mathbf{N}$ such that for every $n \geq N^{\prime}, a_{n}>a$. For every 
$n \geq \max \left\{N, N^{\prime}\right\}$, we have $a_{n}>a$ and $z_{n}<z+\eta$, thus by monotonicity of

$A, A\left(z_{n}, a_{n}\right) \leq A\left(z+\eta, a_{n}\right)<a_{n}$ which contradicts the definition of $a_{n}$, and proves that $a_{n}$ is bounded.

In particular, since $a_{n}$ is bounded, permuting $v_{n}$ and $v_{n}^{\prime}$ if necessary, we have $v_{n}^{\prime} \geq v_{n}>a_{n}$ for $n$ large enough. Equation F.3 gives, for $n$ large enough

(F.4) $\varepsilon \leq\left|A\left(z_{n}, v_{n}^{\prime}\right)-A\left(z_{n}, v_{n}\right)\right| \leq \frac{1}{n}$

a contradiction for $n$ large enough.

\section{G. Proof of Theorem 4.1.}

First prove the Theorem if Assumption i) is true. In this proof, to simplify notations, we let $w=\bar{w}$ and $v^{*}=\bar{v}^{*}$. The proof is similar for $v^{*}=\underline{v}^{*}$. Remark that from Boundary condition and Uniform continuity in $v$, we get that for every $z \in Z$, the mapping $v \in[-\infty,+\infty] \rightarrow A(z, v)$ is continuous: this will be used in this proof. Let $x_{0} \in X$.

Step 1: first show $B v^{*}\left(x_{0}\right) \geq v^{*}\left(x_{0}\right)$.

First case: If $v^{*}\left(x_{0}\right)=-\infty$ then $B v^{*}\left(x_{0}\right) \geq v^{*}\left(x_{0}\right)$ is true.

Second case: $v^{*}\left(x_{0}\right)=+\infty$. Thus, by definition of $v^{*}\left(x_{0}\right)$, for every $K>0$, there exists a strictly increasing sequence $\left(T_{n}\right)$ of integers, and a sequence $\left(z_{n}\right)_{n \geq 0}$ of $\Sigma\left(x_{0}\right)$ such that

$$
\lim _{N \rightarrow+\infty} A^{T_{N}}\left(\left(z_{n}\right)_{n \geq 0}, 0\right) \geq K
$$

Now, by definition of $B v^{*}\left(x_{0}\right)$ and the definition of $v^{*}$, we get in particular

$$
\begin{aligned}
B v^{*}\left(x_{0}\right) & \geq A\left(z_{0}, \varlimsup_{N \rightarrow+\infty} A^{T_{N}-1}\left(\left(z_{n}\right)_{n \geq 1}, 0\right)\right. \\
& \geq \underline{\lim }_{N \rightarrow+\infty} A\left(z_{0}, A^{T_{N}-1}\left(\left(z_{n}\right)_{n \geq 1}, 0\right)\right. \\
& =\lim _{N \rightarrow+\infty} A^{T_{N}}\left(\left(z_{n}\right)_{n \geq 0}, 0\right) \geq K .
\end{aligned}
$$

Here, we apply to the continuous mapping $v \rightarrow A\left(z_{0}, v\right)$ the fact that for every continuous mapping $f, f\left(\overline{\lim }_{n \rightarrow \infty} a_{n}\right) \geq \underline{\lim }_{n \rightarrow \infty} f\left(a_{n}\right)$. 
If $K \rightarrow+\infty$, this gives $B v^{*}\left(x_{0}\right)=+\infty=v^{*}\left(x_{0}\right)$.

Last case: $v^{*}\left(x_{0}\right)$ is finite. Similarly to the case above, this implies that for every $\varepsilon>0$, there exists a strictly increasing sequence $\left(T_{n}\right)$ of integers, and a sequence $\left(z_{n}\right)_{n \geq 0} \in \Sigma^{0}\left(x_{0}\right)$ such that $\lim _{N \rightarrow+\infty} A^{T_{N}}\left(\left(z_{n}\right)_{n \geq 0}, 0\right) \geq v^{*}\left(x_{0}\right)-\varepsilon$. The same proof as above (replacing $K$ by $v^{*}\left(x_{0}\right)-\varepsilon$ ) finally gives

$$
B v^{*}\left(x_{0}\right) \geq v^{*}\left(x_{0}\right)-\varepsilon
$$

for every $\varepsilon>0$. Thus, we get the inequality when $\varepsilon \rightarrow 0$.

Step 2: Now, we show $B v^{*}\left(x_{0}\right) \leq v^{*}\left(x_{0}\right)$.

If $B v^{*}\left(x_{0}\right)=-\infty$ then the inequality is true. Thus, consider the two other cases:

1) First case: $B v^{*}\left(x_{0}\right) \in \mathbf{R}$.

Let $\varepsilon>0$. By definition of $B v^{*}\left(x_{0}\right)$, there exists $x_{1} \in \Gamma\left(x_{0}\right)$ and $z_{0} \in$ $\Omega\left(x_{0}, x_{1}\right)$ such that

$$
B v^{*}\left(x_{0}\right) \leq A\left(z_{0}, v^{*}\left(x_{1}\right)\right)+\varepsilon
$$

We now show that in the three following subcases $\left(v^{*}\left(x_{1}\right)=+\infty, v^{*}\left(x_{1}\right)=\right.$ $-\infty$ and $\left.v^{*}\left(x_{1}\right) \in \mathbf{R}\right)$, we have $v^{*}\left(x_{0}\right) \geq B v^{*}\left(x_{0}\right)-2 \varepsilon$.

First subcase: $v^{*}\left(x_{1}\right)=+\infty$. Thus,

(G.2) $B v^{*}\left(x_{0}\right) \leq A\left(z_{0},+\infty\right)+\varepsilon$.

By definition of $v^{*}\left(x_{1}\right)=+\infty$, for every $K>0$, there exists a strictly increasing sequence $\left(T_{n}\right)$ of integers, and a sequence $\left(z_{n}\right)_{n \geq 1}$ of $\Sigma\left(x_{1}\right)$ such that

$$
\lim _{N \rightarrow+\infty} A^{T_{N}}\left(\left(z_{n}\right)_{n \geq 1}, 0\right) \geq K
$$

From the definition of $v^{*}\left(x_{0}\right)$, we have

$$
\begin{aligned}
v^{*}\left(x_{0}\right) & \geq \varlimsup_{\lim } \rightarrow+\infty \\
& \geq A\left(z_{0}, A^{T_{N}}\left(\left(z_{n}\right)_{n \geq 1}, 0\right)\right) \\
& \geq A\left(z_{0}, K\right)
\end{aligned}
$$


Passing to the limit when $K$ tends to $+\infty$, by Boundary assumption, we get $v^{*}\left(x_{0}\right) \geq A\left(z_{0},+\infty\right)$. From equation G.2, we get

$v^{*}\left(x_{0}\right) \geq B v^{*}\left(x_{0}\right)-\varepsilon$.

Second subcase: $v^{*}\left(x_{1}\right)=-\infty$. Thus,

(G.3) $B v^{*}\left(x_{0}\right) \leq A\left(z_{0},-\infty\right)+\varepsilon$.

By definition of $v^{*}\left(x_{0}\right)$ and Increasing Monotonicity assumption, we have $v^{*}\left(x_{0}\right) \geq A\left(z_{0},-\infty\right)$. From Equation G.3, we get $v^{*}\left(x_{0}\right) \geq B v^{*}\left(x_{0}\right)-\varepsilon$.

Last subcase: $v^{*}\left(x_{1}\right) \in \mathbf{R}$.

Since $A$ is uniformly continuous in $v$, there exists $\eta>0$ (now fixed) such that:

$$
\forall\left(z_{0}, v, v^{\prime}\right) \in Z \times \mathbf{R}^{2},\left|v-v^{\prime}\right| \leq \eta \Rightarrow\left|A\left(z_{0}, v\right)-A\left(z_{0}, v^{\prime}\right)\right| \leq \varepsilon
$$

By definition of $v^{*}\left(x_{1}\right)$, there exists a strictly increasing sequence $\left(T_{n}\right)$ of integers, and a sequence $\left(z_{n}\right)_{n \geq 1}$ of $\Sigma\left(x_{1}\right)$ such that

$$
\lim _{N \rightarrow+\infty} A^{T_{N}}\left(\left(z_{n}\right)_{n \geq 1}, 0\right) \geq v^{*}\left(x_{1}\right)-\eta
$$

From the definition of $v^{*}\left(x_{0}\right)$, we have

$$
\begin{aligned}
v^{*}\left(x_{0}\right) & \geq \varlimsup \overline{\operatorname{im}}_{N \rightarrow+\infty} A\left(z_{0}, A^{T_{N}}\left(\left(z_{n}\right)_{n \geq 1}, 0\right)\right) \\
& \geq A\left(z_{0}, \underline{\lim }_{N \rightarrow+\infty} A\left(z_{0}, A^{T_{N}}\left(\left(z_{n}\right)_{n \geq 1}, 0\right)\right)\right. \\
& \geq A\left(z_{0}, v^{*}\left(x_{1}\right)-\eta\right) \geq A\left(z_{0}, v^{*}\left(x_{1}\right)\right)-\varepsilon,
\end{aligned}
$$

the last inequality being a consequence of Equation G.4. From $B v^{*}\left(x_{0}\right) \leq$ $A\left(z_{0}, v^{*}\left(x_{1}\right)\right)+\varepsilon$, we finally get

$v^{*}\left(x_{0}\right) \geq B v^{*}\left(x_{0}\right)-2 \varepsilon$.

Finally, this inequality still holds in all the subcases above. Passing to the limit when $\varepsilon \rightarrow 0$, we get $v^{*}\left(x_{0}\right) \geq B v^{*}\left(x_{0}\right)$.

2) Second case: $B v^{*}\left(x_{0}\right)=+\infty$.

By definition of $B v^{*}\left(x_{0}\right)$, for every $K>0$, there exists $x_{1} \in \Gamma\left(x_{0}\right)$ and $z_{0} \in \Omega\left(x_{0}, x_{1}\right)$ such that $A\left(z_{0}, v^{*}\left(x_{1}\right)\right) \geq K$. This is exactly Equation G.1, in 
which $B v^{*}\left(x_{0}\right)$ is replaced by $K+\varepsilon$. The same proof as the first case can then be applied formally, with such a modification. This provides $v^{*}\left(x_{0}\right) \geq K-\varepsilon$, which finally give $v^{*}\left(x_{0}\right)=+\infty$ for $K \rightarrow+\infty$. Thus $B v^{*}\left(x_{0}\right)=v^{*}\left(x_{0}\right)=$ $+\infty$.

Now, we prove the theorem when Assumption ii) is true (instead of Assumption i)). The only places where we have used Assumption i) in the proof above is:

- to derive Equation G.4. But from $\Sigma\left(x_{0}\right)$ compact, we get that $z_{0}$ in Equation G.4 is constrained to belong to a compact subset of $Z$, and thus the Equation still holds from Compact uniform continuity assumption in $v$.

- To get that for every $z \in Z$, the mapping $v \in[-\infty,+\infty] \rightarrow A(z, v)$ is continuous, which is clearly true if $A$ is only assumed to be compactly uniformly continuous in $v$ (since continuity at $v=+\infty$ or $v=-\infty$ is a consequence of Boundary assumption).

Thus, the proof is unchanged when Assumption ii) is assumed.

\section{H. Proof of Proposition 4.3}

Let $\delta$ be such that for every $\left(z, v, v^{\prime}\right) \in Z \times \mathbf{R}^{2},\left|A(z, v)-A\left(z, v^{\prime}\right)\right| \leq \delta\left(v-v^{\prime}\right)$, where $\delta: \mathbf{R} \rightarrow \mathbf{R} \cup\{+\infty\}$ tends to 0 at 0 . Existence of $\delta$ follows from Lemma 4.1. Let $x_{0} \in X$. For every $(\underset{\sim}{z}, \underset{\sim}{x}) \in \widetilde{\Sigma}\left(x_{0}\right)$, by iteration and by Increasing Monotonicity, one obtains:

$$
\begin{gathered}
\left.A\left(z_{0}, A\left(z_{1}, A\left(z_{2}, \ldots, A\left(z_{n-1}, \bar{v}\left(x_{n}\right)\right)+\varepsilon_{n-1}\right)+\varepsilon_{n-2}+\cdots\right)+\varepsilon_{2}\right)+\varepsilon_{1}\right) \\
\leq A\left(z_{0}, A\left(z_{1}, A\left(z_{2}, \ldots, A\left(z_{n-1}, \bar{v}\left(x_{n}\right)\right)\right)+\cdots\right)\right) \\
\cdots+\delta\left(\varepsilon_{1}+\delta\left(\varepsilon_{2}+\cdots+\delta\left(\varepsilon_{n-2}+\delta\left(\varepsilon_{n-1}\right)\right) \cdots\right) .\right.
\end{gathered}
$$

For every $a>0$, define

$$
\begin{aligned}
& V_{a}=\left\{\left(\varepsilon_{n}\right)_{n \in \mathbf{N}^{*}} \in[0,1]^{\mathbf{N}}: \forall\left(\varepsilon_{n}^{\prime}\right)_{n \in \mathbf{N}^{*}}\right. \in \prod_{i \in \mathbf{N}^{*}}\left[0,2 \varepsilon_{i}\right] \\
&\left.\delta\left(\varepsilon_{1}^{\prime}\right) \leq a, \delta\left(\varepsilon_{2}^{\prime}\right) \leq \varepsilon_{1}, \ldots, \delta\left(\varepsilon_{n-1}^{\prime}\right) \leq \varepsilon_{n-2}, \ldots\right\}
\end{aligned}
$$


This set is nonempty (it contains 0 ). Moreover, every $V_{a}$ intersects $\left.] 0,1\right]^{\mathbf{N}}$ (indeed, since $\delta$ is continuous at 0 , one can define some $\left.\left.\left(\varepsilon_{n}\right)_{n \in \mathbf{N}^{*}} \in V_{a} \cap\right] 0,1\right]^{\mathbf{N}}$ inductively). Consider on $[0,1]^{\mathbf{N}}$ the topology generated by this family of neighbourhood of 0 . Then every neighborhood of 0 intersects $] 0,1]^{\mathbf{N}}$. Moreover, for every $\left(\varepsilon_{n}\right)_{n \in \mathbf{N}^{*}} \in V_{a}$ and every integer $n \geq 1, \varepsilon_{n-2}+\delta\left(\varepsilon_{n-1}\right) \leq \varepsilon_{n-2}+\varepsilon_{n-2}$, thus $\delta\left(\varepsilon_{n-2}+\delta\left(\varepsilon_{n-1}\right)\right) \leq \varepsilon_{n-3}$. Iterating, one gets

$$
\forall n \in \mathbf{N}, \delta\left(\varepsilon_{1}+\delta\left(\varepsilon_{2}+\cdots+\delta\left(\varepsilon_{n-2}+\delta\left(\varepsilon_{n-1}\right)\right) \cdots\right) \leq a\right.
$$

From Equation (H.1) and the definition of $f$, taking the infimum with respect to $n$, and then the supremum, one obtains:

$$
\forall \varepsilon \in V_{a}, f(\varepsilon) \leq f(0)+a
$$

which proves the weak continuity of $A$, that concludes the proof.

\section{Proof of Proposition 4.4}

Let $x_{0} \in X$. Since $\Sigma\left(x_{0}\right)$ is compact (for the product topology), for every $n \geq 0$ there exists $K_{n}$ a compact subset of $Z$ such that for every $(\underset{\sim}{z} \underset{\sim}{x}) \in \widetilde{\Sigma}\left(x_{0}\right)$ (which implies $z \in \Sigma\left(x_{0}\right)$ ), we have $z_{n} \in K_{n}$ for every $n \geq 0$.

For every $n \geq 0$, from compact uniform continuity in $v$, there exists $\delta_{n}$ such that for every $\left(z, v, v^{\prime}\right) \in K_{n} \times \mathbf{R}^{2},\left|A(z, v)-A\left(z, v^{\prime}\right)\right| \leq \delta_{n}\left(v-v^{\prime}\right)$, where $\delta_{n}: \mathbf{R} \rightarrow \mathbf{R} \cup\{+\infty\}$ tends to 0 at 0 . For every $(\underset{\sim}{z}, \underset{\sim}{x}) \in \widetilde{\Sigma}\left(x_{0}\right)$, by iteration and by Increasing Monotonicity, one obtains:

$$
\begin{aligned}
& \left.A\left(z_{0}, A\left(z_{1}, A\left(z_{2}, \ldots, A\left(z_{n-1}, \bar{v}\left(x_{n}\right)\right)+\varepsilon_{n-1}\right)+\varepsilon_{n-2}+\cdots\right)+\varepsilon_{2}\right)+\varepsilon_{1}\right) \\
& \leq A\left(z_{0}, A\left(z_{1}, A\left(z_{2}, \ldots, A\left(z_{n-1}, \bar{v}\left(x_{n}\right)\right)\right)+\cdots\right)\right) \\
& \cdots+\delta_{0}\left(\varepsilon_{1}+\delta_{1}\left(\varepsilon_{2}+\cdots+\delta_{n-3}\left(\varepsilon_{n-2}+\delta_{n-2}\left(\varepsilon_{n-1}\right)\right) \cdots\right) .\right.
\end{aligned}
$$

For every $a>0$, define

$$
\begin{aligned}
V_{a}=\left\{\left(\varepsilon_{n}\right)_{n \in \mathbf{N}^{*}}\right. & \in[0,1]^{\mathbf{N}}: \forall\left(\varepsilon_{n}^{\prime}\right)_{n \in \mathbf{N}^{*}} \in \prod_{i \in \mathbf{N}^{*}}\left[0,2 \varepsilon_{i}\right] \\
& \left.\delta_{0}\left(\varepsilon_{1}^{\prime}\right) \leq a, \delta_{1}\left(\varepsilon_{2}^{\prime}\right) \leq \varepsilon_{1}, \ldots, \delta_{n-2}\left(\varepsilon_{n-1}^{\prime}\right) \leq \varepsilon_{n-2}, \ldots\right\}
\end{aligned}
$$

The end of the proof is similar to the proof of Proposition 4.3. 


\section{J. Proof of Theorem 4.2}

Consider $[\underline{v}, \bar{v}]$ such that $(\underline{v}, \bar{v}) \in V^{2}$ satisfies Transversality, and let $v$ be a fixed point of $B$ on $[\underline{v}, \bar{v}]$, with $\bar{v}\left(x_{0}\right)<+\infty$ for every $x_{0} \in X$.

Step 1. First prove that $v \geq \bar{v}^{*}$.

Let $x_{0} \in X$.

If $\bar{v}^{*}\left(x_{0}\right)=-\infty$ then $v\left(x_{0}\right) \geq \bar{v}^{*}\left(x_{0}\right)$. Assume now $\bar{v}^{*}\left(x_{0}\right)>-\infty$. Then $\bar{v}^{*}\left(x_{0}\right)=\sup _{z \in \Sigma\left(x_{0}\right)} \bar{w}(z)=\sup _{z \in \Sigma^{0}\left(x_{0}\right)} \bar{w}(z)$, where $\Sigma^{0}\left(x_{0}\right)=\left\{z \in \Sigma\left(x_{0}\right):\right.$ $\bar{w}(z)>-\infty\}$.

By definition of $B$ and since $v$ is a fixed point of $B$, one has

$$
\forall x_{1} \in \Gamma\left(x_{0}\right), \forall z_{0} \in \Omega\left(x_{0}, x_{1}\right), v\left(x_{0}\right)=B v\left(x_{0}\right) \geq A\left(z_{0}, v\left(x_{1}\right)\right)
$$

Similarly,

$$
\forall x_{2} \in \Gamma\left(x_{1}\right), \forall z_{1} \in \Omega\left(x_{1}, x_{2}\right), v\left(x_{1}\right)=B v\left(x_{1}\right) \geq A\left(z_{1}, v\left(x_{2}\right)\right)
$$

and then, from Increasing Monotonicity, for every $x_{1} \in \Gamma\left(x_{0}\right), x_{2} \in \Gamma\left(x_{1}\right), z_{0} \in$ $\Omega\left(x_{0}, x_{1}\right), z_{1} \in \Omega\left(x_{1}, x_{2}\right)$, one has:

$$
B v\left(x_{0}\right) \geq A\left(z_{0}, A\left(z_{1}, v\left(x_{2}\right)\right)\right)
$$

And by induction,

$$
\forall T \in \mathbf{N}^{*}, \forall(\underset{\sim}{z}, \underset{\sim}{x}) \in \widetilde{\Sigma}\left(x_{0}\right), B v\left(x_{0}\right) \geq A^{T}\left(\underset{\sim}{z}, v\left(x_{T}\right)\right)
$$

Since $v \geq \underline{v}$ and by Increasing Monotonicity,

$$
\forall T \in \mathbf{N}^{*}, \forall(\underset{\sim}{z}, \underset{\sim}{x}) \in \widetilde{\Sigma}\left(x_{0}\right), B v\left(x_{0}\right) \geq A^{T}\left(\underset{z}{z}, \underline{v}\left(x_{T}\right)\right)
$$

If there exists $T \in \mathbf{N}^{*}$ such that $A^{T}\left(z, \underline{v}\left(x_{T}\right)\right)=+\infty$, then $v\left(x_{0}\right)=B v\left(x_{0}\right)=$ $+\infty \geq \bar{v}^{*}\left(x_{0}\right)$.

Assume now that for every integer $T \in \mathbf{N}^{*}, A^{T}\left(\underset{z}{z} \underline{v}\left(x_{T}\right)\right)<+\infty$. Since $\underline{v}$ satisfies Transversality assumption (T1), this implies $A^{T}(z, 0)<+\infty$ for every integer $T$ large enough. Let $\underset{\sim}{z} \underset{\sim}{x}) \in \widetilde{\Sigma}\left(x_{0}\right)$. Now, we prove that $B v\left(x_{0}\right) \geq \bar{w}(z)$. If $\bar{w}(z)=-\infty$, then it is true. Now assume $\bar{w}(z)>-\infty$, that is $\underset{\sim}{z} \underset{\sim}{x}) \in \widetilde{\Sigma}^{0}\left(x_{0}\right)$. Then $A^{T}(\underset{\sim}{z}, 0)>-\infty$ (thus $A^{T}(z, 0)$ is real) for an infinite number of $T$. From Equation J.5 and for an infinite number of $T$ :

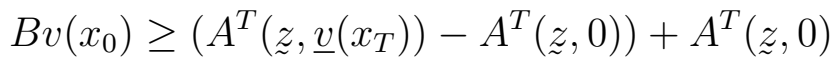


Taking the supremum limit, Transversality Assumption (T1) implies

$$
B v\left(x_{0}\right) \geq \underline{\lim }_{T \rightarrow \infty}\left(A^{T}\left(z, \underline{v}\left(x_{T}\right)-A^{T}(z, 0)\right)+\varlimsup_{\bar{z}} A_{T} A^{T}(z, 0)=\bar{w}(z)\right.
$$

So, for every $z \in \Sigma\left(x_{0}\right), B v\left(x_{0}\right) \geq \bar{w}(z)$.

Taking the supremum on $\Sigma\left(x_{0}\right)$,

$(\mathrm{J} .8) \quad v\left(x_{0}\right)=B v\left(x_{0}\right) \geq \bar{v}^{*}\left(x_{0}\right)$.

Step 2. Let us prove that $v \leq \underline{v}^{*}$.

Let $x_{0} \in X$. If $v\left(x_{0}\right)=-\infty$, then $v\left(x_{0}\right) \leq \underline{v}^{*}\left(x_{0}\right)$. Since $v\left(x_{0}\right) \leq \bar{v}\left(x_{0}\right)<$ $+\infty$, assume now $v\left(x_{0}\right) \in \mathbf{R}$.

For every integer $n$, let $\varepsilon_{n}>0$. From the definition of $B v$, there exists $x_{1} \in \Gamma\left(x_{0}\right)$ and $z_{0} \in \Omega\left(x_{0}, x_{1}\right)$ (depending on $\left.\varepsilon_{1}\right)$ such that

$$
v\left(x_{0}\right)=B v\left(x_{0}\right) \leq A\left(z_{0}, v\left(x_{1}\right)\right)+\varepsilon_{1}
$$

where $v\left(x_{1}\right) \leq \bar{v}\left(x_{1}\right)<+\infty$ by assumption.

Similarly, there exists $x_{2} \in \Gamma\left(x_{1}\right)$ and $z_{1} \in \Omega\left(x_{1}, x_{2}\right)$ (depending on $\varepsilon_{1}$ and $\left.\varepsilon_{2}\right)$ such that

$(\mathrm{J} .10) v\left(x_{1}\right) \leq A\left(z_{1}, v\left(x_{2}\right)\right)+\varepsilon_{2}$.

Then by Increasing Monotonicity,

$$
v\left(x_{0}\right) \leq A\left(z_{0}, A\left(z_{1}, v\left(x_{2}\right)\right)+\varepsilon_{2}\right)+\varepsilon_{1}
$$

By induction, one builds $\underset{\sim}{z} \underset{\sim}{x}) \in \widetilde{\Sigma}\left(x_{0}\right)$ such that

$$
\begin{array}{r}
\forall n \in \mathbf{N}^{*}, v\left(x_{0}\right) \leq A\left(z_{0}, A\left(z_{1}, A\left(z_{2}, \ldots, A\left(z_{n-1}, \bar{v}\left(x_{n}\right)\right)+\varepsilon_{n}\right)\right.\right. \\
\left.\left.+\varepsilon_{n-1}+\cdots\right)+\varepsilon_{2}\right)+\varepsilon_{1} .
\end{array}
$$

by $v \leq \bar{v}<+\infty$ and Increasing Monotonicity Assumption.

Take the infimum with respect to $n$ and then the supremum with respect to $(\underset{\sim}{z}, \underset{\sim}{x}) \in \widetilde{\Sigma}\left(x_{0}\right)$,

$$
\begin{array}{r}
v\left(x_{0}\right) \leq \sup _{(z, x) \in \widetilde{\Sigma}\left(x_{0}\right)} \inf _{n \geq 1} A\left(z_{0}, A\left(z_{1}, A\left(z_{2}, \ldots, A\left(z_{n-1}, \bar{v}\left(x_{n}\right)\right)+\varepsilon_{n}\right)\right.\right. \\
\left.\left.+\varepsilon_{n-1}+\cdots\right)+\varepsilon_{2}\right)+\varepsilon_{1} .
\end{array}
$$


Then, passing to the limit when $\left(\varepsilon_{n}\right)_{n \geq 0}$ tends to 0 , by Weak continuity,

$(\mathrm{J} .13) v\left(x_{0}\right) \leq \sup _{(z, x) \in \widetilde{\Sigma}\left(x_{0}\right)} \inf _{n \geq 1} A\left(z_{0}, A\left(z_{1}, A\left(z_{2}, \ldots, A\left(z_{n-1}, \bar{v}\left(x_{n}\right)\right)\right) \cdots\right)\right)$.

Fix $\varepsilon>0$. From Equation J.13, the supremum being finite or infinite, there exists $(\underset{\sim}{z} \underset{\sim}{x}) \in \widetilde{\Sigma}\left(x_{0}\right)$ such that for every integer $n \geq 1$,

$(\mathrm{J} .14) v\left(x_{0}\right) \leq A^{n}\left(z, \bar{v}\left(x_{n}\right)\right)+\varepsilon$.

Now, $v\left(x_{0}\right)>-\infty$ implies $A^{n}\left(z, \bar{v}\left(x_{n}\right)\right)>-\infty$ for every $n \geq 1$, and by Transversality Assumption (T2), $A^{n}(z, 0)>-\infty$ for $n$ large enough.

If $A^{n}(z, 0)=+\infty$ for $n$ large enough, then $v\left(x_{0}\right) \leq \underline{\lim }_{n \rightarrow+\infty} A^{n}(z, 0)=+\infty$, and passing to the supremum, we get $v\left(x_{0}\right) \leq \underline{v}^{*}\left(x_{0}\right)$, which ends the proof in this case.

Thus, we can assume that $I=\left\{n \geq 0: A^{n}(\underset{z}{z}, 0)<+\infty\right\}$ is infinite. In particular, from above, for $n \in I$ large enough, $A^{n}(z, 0)$ is a real. From (T2), we have $A^{n}\left(z, \bar{v}\left(x_{n}\right)\right)-A^{n}(z, 0) \leq \varepsilon$ for $n$ large enough.

Then, from Equation J.14, for $n \in I$ large enough,

$(\mathrm{J} .15) v\left(x_{0}\right) \leq\left(A^{n}\left(\underset{z}{z}, \bar{v}\left(x_{n}\right)\right)-A^{n}(\underset{\sim}{z}, 0)\right)+A^{n}(\underset{\sim}{z}, 0)+\varepsilon \leq A^{n}(\underset{\sim}{z}, 0)+2 \varepsilon$.

If $A^{n}(z, 0)=+\infty$ (i.e. $\left.n \notin I\right), v\left(x_{0}\right) \leq A^{n}(\underset{z}{z}, 0)+2 \varepsilon$ is also satisfied, and it is thus satisfied for every $n$ large enough.

This implies $v\left(x_{0}\right) \leq \underline{v}^{*}\left(x_{0}\right)$ (take the infimum limit with respect to $n$, majorize by the supremum with respect to $\underset{\sim}{z} \Sigma\left(x_{0}\right)$, and finally take the limit when $\varepsilon \rightarrow 0)$.

Thus, this ends the proof of Assertion 1.a. in Theorem 4.2

Step 3. Let us prove Assertion 1.b. in Theorem 4.2. Let $f \in V$ such that $f \leq v$ and $f$ satisfies (T1). Recall that $v=\underline{v}^{*}=\bar{v}^{*}$.

Since $f \leq v$, since $B$ is non-decreasing and $B v=v, B^{n} f \leq v$, and for every $x_{0} \in X$,

(J.16) $\varlimsup_{n \rightarrow+\infty} B^{n} f\left(x_{0}\right) \leq v\left(x_{0}\right)$

From the definition of $B$, for any $x_{0} \in X$ :

(J.17) $\forall x_{1} \in \Gamma\left(x_{0}\right), \forall z_{0} \in \Omega\left(x_{0}, x_{1}\right), B^{2} f\left(x_{0}\right) \geq A\left(z_{0}, B f\left(x_{1}\right)\right)$. 
Similarly,

(J.18) $\forall x_{2} \in \Gamma\left(x_{1}\right), \forall z_{1} \in \Omega\left(x_{1}, x_{2}\right), B f\left(x_{1}\right) \geq A\left(z_{1}, f\left(x_{2}\right)\right)$.

By Increasing Monotonicity, for every $x_{1} \in \Gamma\left(x_{0}\right), x_{2} \in \Gamma\left(x_{1}\right), z_{0} \in \Omega\left(x_{0}, x_{1}\right)$ and $z_{1} \in \Omega\left(x_{1}, x_{2}\right)$, one has

$$
B^{2} f\left(x_{0}\right) \geq A\left(z_{0}, A\left(z_{1}, f\left(x_{2}\right)\right)\right)
$$

And by induction,

(J.20) $\forall n \in \mathbf{N}^{*}, \forall(\underset{\sim}{z} \underset{\sim}{x}) \in \widetilde{\Sigma}\left(x_{0}\right), B^{n} f\left(x_{0}\right) \geq A^{n}\left(\underset{\sim}{z}, f\left(x_{n}\right)\right)$.

Take $(\underset{\sim}{z}, \underset{\sim}{x}) \in \widetilde{\Sigma}\left(x_{0}\right)$.

Since $v\left(x_{0}\right)=\bar{v}^{*}\left(x_{0}\right)<+\infty, \varlimsup_{\lim _{n \rightarrow+\infty}} A^{n}(z, 0)<+\infty$, thus $A^{n}(z, 0)<+\infty$ for $n$ large enough.

If $v\left(x_{0}\right)=-\infty, \varlimsup_{n \rightarrow+\infty} B^{n} f\left(x_{0}\right) \geq v\left(x_{0}\right)$, and together with Equation J.16, $\varlimsup_{n \rightarrow+\infty} B^{n} f\left(x_{0}\right)=v\left(x_{0}\right)$ is proved in this case.

Assume now that $v\left(x_{0}\right)>-\infty$, and let us prove that for every $z \in \Sigma^{0}\left(x_{0}\right)$

(J.21) $\varlimsup_{n \rightarrow+\infty} B^{n} f\left(x_{0}\right) \geq \underline{\lim }_{n \rightarrow+\infty} A^{n}(z, 0)$.

If $A^{n}(z, 0)=-\infty$ for an infinite number of $n, \underline{\lim }_{n \rightarrow+\infty} A^{n}(\underset{z}{z}, 0)=-\infty$ and Equation J.21 is true.

Otherwise, if $A^{n}(z, 0)>-\infty$ for $n$ large enough, from above, we can assume that $A^{n}(z, 0)$ is finite for $n$ large enough, and from Equation J.20

$$
B^{n} f\left(x_{0}\right) \geq\left(A^{n}\left(\underset{z}{z}, f\left(x_{n}\right)\right)-A^{n}(\underset{z}{z}, 0)\right)+A^{n}(\underset{z}{z}, 0)
$$

for $n$ large enough. Taking then the supremum limit, we get

$$
\varlimsup_{n \rightarrow+\infty} B^{n} f\left(x_{0}\right) \geq \underline{\lim }_{n \rightarrow+\infty}\left(A^{n}\left(z, f\left(x_{n}\right)\right)-A^{n}(z, 0)\right)+\underline{\lim }_{n \rightarrow+\infty} A^{n}(z, 0) .
$$

Thus from (T1) Assumption satisfied by $f$, for every $z \in \Sigma^{0}\left(x_{0}\right)$ we get Equation J.21, which is also true when $z \notin \Sigma^{0}\left(x_{0}\right)$, because then $\underline{\lim }_{n \rightarrow+\infty} A^{n}(z, 0)=$ $-\infty$

Taking the supremum for $\underset{\sim}{z} \in \Sigma\left(x_{0}\right)$ in J.21, we finally get $\varlimsup_{n \rightarrow+\infty} B^{n} f\left(x_{0}\right) \geq$ $\underline{v}^{*}\left(x_{0}\right)=v\left(x_{0}\right)$, and from Equation J.16, Assertion 1.b is proved. 
Step 4. We finally prove Assertion 2. Assume $\underline{v}$ and $\bar{v}$ above also satisfy $\underline{v} \leq B \underline{v}$ and $B \bar{v} \leq \bar{v}$, then, from Tarski fixed point theorem on $[\underline{v}, \bar{v}], B$ admits a fixed-point on $[\underline{v}, \bar{v}]$, and from the first part of Theorem 4.2, this fixed-point is equal to the value function.

\section{K. Another Definition of Biconvergence}

To prove the equivalence between the two definitions, first assume $U$ is a $A$ recursive payoff satisfying definition 4.4 of biconvergence (the original definition of Streufert). Take $\underset{\sim}{x} \in \prod_{t=1}^{+\infty} \Gamma^{t}\left(x_{0}\right)$ and $\varepsilon>0$. By upper convergence, there exists an integer $N_{1}>0$ such that for every $N \geq N_{1}$,

$$
\left.\sup U\left(x_{1}, \ldots, x_{N}, \prod_{s=N+1}^{+\infty} \Gamma^{s}\left(x_{0}\right)\right)\right) \leq U(\underset{\sim}{x})+\varepsilon,
$$

thus for every $\left(x_{N+1}^{\prime}, x_{N+2}^{\prime}, \ldots\right) \in \prod_{t=N+1}^{+\infty} \Gamma^{t}\left(x_{0}\right)$,

$$
U\left(x_{1}, x_{2}, \ldots, x_{N}, x_{N+1}^{\prime}, x_{N+2}^{\prime}, \ldots\right)-U\left(x_{1}, x_{2}, \ldots, x_{N}, x_{N+1}, x_{N+2}, \ldots\right) \leq \varepsilon .
$$

Similarly, lower convergence implies that there exists an integer $N_{2}>0$ such that for every $N \geq N_{2}$ and every $\left(x_{N+1}^{\prime}, x_{N+2}^{\prime}, \ldots\right) \in \prod_{t=N+1}^{+\infty} \Gamma^{t}\left(x_{0}\right)$, one has:

$U\left(x_{1}, x_{2}, \ldots, x_{N}, x_{N+1}^{\prime}, x_{N+2}^{\prime}, \ldots\right)-U\left(x_{1}, x_{2}, \ldots, x_{N}, x_{N+1}, x_{N+2}, \ldots\right) \geq-\varepsilon$

Then take $N=\max \left\{N_{1}, N_{2}\right\}$ to get the other definition.

For the conversely statement, take $\underset{\sim}{x} \in \prod_{t=1}^{+\infty} \Gamma^{t}\left(x_{0}\right), \varepsilon>0$ and assume there exists an integer $N^{\prime}>0$ such that for every $N \geq N^{\prime}$ and for every $\left(x_{N+1}^{\prime}, x_{N+2}^{\prime}, \ldots\right) \in \prod_{t=N+1}^{+\infty} \Gamma^{t}\left(x_{0}\right)$, one has:

$$
\left|U\left(x_{1}, x_{2}, \ldots, x_{N}, x_{N+1}, x_{N+2}, \ldots\right)-U\left(x_{1}, x_{2}, \ldots, x_{N}, x_{N+1}^{\prime}, x_{N+2}^{\prime}, \ldots\right)\right| \leq \frac{\varepsilon}{2} .
$$

By definition,

$$
\sup U\left(x_{1}, \ldots, x_{N}, \prod_{s=N+1}^{+\infty} \Gamma^{s}\left(x_{0}\right)\right) \geq U(\underset{\sim}{x})
$$

because $\left(x_{N+1}, x_{N+2}, \ldots\right) \in \prod_{s=N+1}^{+\infty} \Gamma^{s}\left(x_{0}\right)$. In addition, there exists $\left(x_{N+1}^{\prime}, x_{N+2}^{\prime}, \ldots\right) \in$ $\prod_{s=N+1}^{+\infty} \Gamma^{s}\left(x_{0}\right)$ such that

$$
U\left(x_{1}, x_{2}, \ldots, x_{N}, x_{N+1}^{\prime}, x_{N+2}^{\prime}, \ldots\right) \geq \sup U\left(x_{1}, \ldots, x_{N}, \prod_{s=N+1}^{+\infty} \Gamma^{s}\left(x_{0}\right)\right)-\frac{\varepsilon}{2}
$$


From Equation K.1, we get

$$
U\left(x_{1}, x_{2}, \ldots, x_{N}, x_{N+1}, x_{N+2}, \ldots\right) \geq \sup U\left(x_{1}, \ldots, x_{N}, \prod_{s=N+1}^{+\infty} \Gamma^{s}\left(x_{0}\right)\right)-\varepsilon
$$

Finally, for every $N \geq N^{\prime}$,

$$
\left|U\left(x_{1}, x_{2}, \ldots, x_{N}, x_{N+1}, x_{N+2}, \ldots\right)-\sup U\left(x_{1}, \ldots, x_{N}, \prod_{s=N+1}^{+\infty} \Gamma^{s}\left(x_{0}\right)\right)\right| \leq \varepsilon
$$

and we get upper convergence. Similarly, we get lower convergence.

\section{Proof of Corollary 4.1}

First prove the following Lemma, which provide candidates satisfying Transversality Assumption.

Lemma L.1. Assume Null Assumption, and let $U$ be biconvergent over $\prod_{t=1}^{+\infty} \Gamma^{t}\left(x_{0}\right)$, with $\Gamma$ being compact-valued. Then:

(i) If $A$ is upper semicontinuous, then $\underline{v}\left(x_{0}\right):=\inf _{\underset{\sim}{y \in \prod_{t=1}^{+\infty}} \Gamma^{t}\left(x_{0}\right)} U(\underset{\sim}{y})$ satisfies Transversality Assumption $\left(T_{1}\right)$.

(ii) If $A$ is lower semicontinuous, then $\bar{v}\left(x_{0}\right):=\sup _{\underset{\sim}{y \in \prod_{t=1}^{+\infty} \Gamma^{t}\left(x_{0}\right)}} U(\underset{\sim}{y})$ satisfies Transversality Assumption $\left(T_{2}\right)$.

(iii) For any aggregator $A$, if $\inf _{\underset{\sim}{y \in \prod_{t=1}^{+\infty} \Gamma^{t}\left(x_{0}\right)}} U(\underset{\sim}{y})=\min _{\underset{\sim}{y \in \prod_{t=1}^{+\infty} \Gamma^{t}\left(x_{0}\right)}} U(\underset{\sim}{y})$, then $\underline{v}$ satisfies Transversality Assumption $\left(T_{1}\right)$.

Similarly, if $\sup _{\underset{\sim}{y} \in \prod_{t=1}^{+\infty} \Gamma^{t}\left(x_{0}\right)} U(\underset{\sim}{y})=\max _{\underset{\sim}{y} \in \prod_{t=1}^{+\infty} \Gamma^{t}\left(x_{0}\right)} U(\underset{\sim}{y})$, then $\bar{v}$ satisfies Transversality Assumption $\left(T_{2}\right)$.

To prove this lemma, we use the following Claim:

Claim. (i) If $f:[-\infty,+\infty] \rightarrow[-\infty,+\infty]$ is an upper semicontinuous realvalued function and $g$ is a real-valued function on a metric compact space $M$, then

$$
f\left(\inf _{x \in M} g(x)\right) \geq \inf _{x \in M} f(g(x))
$$


(ii) If $f:[-\infty,+\infty] \rightarrow[-\infty,+\infty]$ is a lower semicontinuous real-valued function and $g$ a real-valued function on a metric compact space $M$, then

$$
f\left(\sup _{x \in M} g(x)\right) \leq \sup _{x \in M} f(g(x))
$$

Proof. By definition, $\inf _{x \in M} g(x)=\lim _{n \rightarrow+\infty} g\left(x_{n}\right)$ for some sequence $\left(x_{n}\right)$ in $M$. Without any loss of generality, since $M$ is compact, one can assume that $\left(x_{n}\right)$ converges to some $x \in M$. Since $f$ is upper semi-continuous,

$$
f\left(\inf _{x \in M} g(x)\right)=f\left(\lim _{n \rightarrow+\infty} g\left(x_{n}\right)\right) \geq \varlimsup_{\lim } \rightarrow+\infty f\left(g\left(x_{n}\right)\right) \geq \inf _{x \in M} f(g(x)) .
$$

The proof is similar for ii).

QED

Now, we prove Lemma L.1, part (i). Let $z \in \Sigma\left(x_{0}\right)$. One has

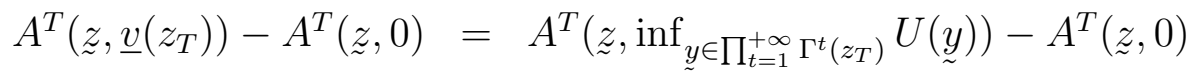

$$
\begin{aligned}
& \text { (by definition of } \underline{v} \text { ) } \\
& \geq \inf _{\underset{y}{y} \prod_{t=1}^{+\infty} \Gamma^{t}\left(z_{T}\right)} A^{T}(\underset{\sim}{z}, U(\underset{\sim}{y}))-A^{T}(\underset{\sim}{z}, 0) \\
& \text { (by Claim i) above) } \\
& =\inf _{\underline{y} \in \prod_{t=1}^{+\infty} \Gamma^{t}\left(z_{T}\right)} A^{T}(\underset{z}{z}, U(\underline{y}))-A^{T}(\underset{z}{z}, U(\underline{a}, \underline{a}, \ldots)) \\
& \text { (by Null assumption) } \\
& =\inf _{\underset{\sim}{y \in \prod_{t=1}^{+\infty} \Gamma^{t}\left(z_{T}\right)}} U\left(z_{1}, \ldots, z_{T}, \underset{\sim}{y}\right)-U\left(z_{1}, \ldots, z_{T}, \underline{a}, \underline{a}, \ldots\right)
\end{aligned}
$$

(since $U$ is $A$-recursive)

This last quantity can be made as small as one wishes for $T$ large enough by biconvergence, which proves that $\underline{v}$ satisfies $\left(T_{1}\right)$.

The proof of Lemma L.1, Part (ii) is similar.

Let us prove Lemma L.1, Part (iii). To show that $\underline{v}$ satisfies $\left(T_{1}\right)$, follow the proof above. Simply note that $\underline{v}\left(x_{T}\right)$ can be written $U(\underset{\sim}{y})$ for some $\underset{\sim}{y}$ (depending on $T$ ), and we can mimic the proof above without using any infimum. This ends the proof of Lemma L.1 (since the proof is similar for $\bar{v})$

We finally prove Corollary 4.1 .

Define, for every $x_{0} \in X, \bar{v}\left(x_{0}\right)=\max U\left(\prod_{t=1}^{+\infty} \Gamma^{t}\left(x_{0}\right)\right)$, and define $\underline{v}\left(x_{0}\right)=$ $\inf U\left(\prod_{t=1}^{+\infty} \Gamma^{t}\left(x_{0}\right)\right)$. Let us show that one can apply Theorem 4.2 to prove that $v^{*}$ is the unique solution to the Bellman equation. 
First, $\underline{v} \leq \bar{v}<+\infty$ (by assumption), and, by definition, for every $\underset{\sim}{x} \in \Sigma\left(x_{0}\right)$, we have $U(\underset{\sim}{x}) \in\left[\underline{v}\left(x_{0}\right), \bar{v}\left(x_{0}\right)\right]$. By Lemma L.1, $(\underline{v}, \bar{v})$ satisfies Transversality Assumption. By Proposition 3.2, $\bar{v}^{*}\left(x_{0}\right)=\underline{v}^{*}\left(x_{0}\right)=\sup _{x \in \Sigma\left(x_{0}\right)} U(\underset{\sim}{x})$.

We shall prove that $B \bar{v} \leq \bar{v}$, and $B \underline{v} \geq \underline{v}$. First, to prove $B \bar{v} \leq \bar{v}$, let $x_{0} \in X$.

$$
\begin{aligned}
B \bar{v}\left(x_{0}\right) & =\sup _{x_{1} \in \Gamma\left(x_{0}\right)} A\left(x_{1}, \bar{v}\left(x_{1}\right)\right)=\sup _{x_{1} \in \Gamma\left(x_{0}\right)} A\left(x_{1}, \max U\left(\prod_{t=1}^{+\infty} \Gamma^{t}\left(x_{1}\right)\right)\right) \\
& =\sup _{x_{1} \in \Gamma\left(x_{0}\right)} \sup _{x_{t+1} \in \Gamma^{t}\left(x_{1}\right), \forall t \geq 1} A\left(x_{1}, U\left(x_{2}, x_{3}, \ldots, x_{n}, \ldots\right)\right) \\
& =\sup _{x_{1} \in \Gamma\left(x_{0}\right)} \sup _{x_{t+1} \in \Gamma^{t}\left(x_{1}\right), \forall t \geq 1} U\left(x_{1}, x_{2}, \ldots, x_{n}, \ldots\right) \\
& \leq \sup _{x_{t} \in \Gamma^{t}\left(x_{0}\right), \forall t \geq 1} U\left(x_{1}, x_{2}, \ldots, x_{n}, \ldots\right)=\bar{v}\left(x_{0}\right)
\end{aligned}
$$

Let us show that $B \underline{v} \geq \underline{v}$.

$$
\begin{aligned}
B \underline{v}\left(x_{0}\right) & =\sup _{x_{1} \in \Gamma\left(x_{0}\right)} A\left(x_{1}, \underline{v}\left(x_{1}\right)\right)=\sup _{x_{1} \in \Gamma\left(x_{0}\right)} A\left(x_{1}, \inf U\left(\prod_{t=1}^{+\infty} \Gamma^{t}\left(x_{1}\right)\right)\right) \\
& \geq \sup _{x_{1} \in \Gamma\left(x_{0}\right)} \inf _{\left(x_{2}, \ldots\right) \in \prod_{t=1}^{+\infty} \Gamma^{t}\left(x_{1}\right)} A\left(x_{1}, U\left(x_{2}, x_{3}, \ldots\right)\right)
\end{aligned}
$$

(from the Claim above)

$$
\geq \sup _{x_{1} \in \Gamma\left(x_{0}\right)} \inf _{\left(x_{2}, \ldots\right) \in \prod_{t=1}^{+\infty} \Gamma^{t}\left(x_{1}\right)} U\left(x_{1}, x_{2}, x_{3}, \ldots\right) \geq \underline{v}\left(x_{0}\right)
$$

To be able to apply Theorem 4.2 , we have finally to prove that $A$ is weakly continuous at $\bar{v}$, i.e. that

$$
\begin{aligned}
f(\varepsilon)=\sup _{(z, x) \in \widetilde{\Sigma}\left(x_{0}\right)} & \inf _{n \geq 0} A\left(z_{0}, A\left(z_{1}, A\left(z_{2}, \ldots\right.\right.\right. \\
& \left.\left.\left.\left.\ldots, A\left(z_{n-1}, \bar{v}\left(x_{n}\right)\right)+\varepsilon_{n-1}\right)+\varepsilon_{n-2}+\cdots\right)+\varepsilon_{2}\right)+\varepsilon_{1}\right)
\end{aligned}
$$

is upper semicontinuous with respect to the sequence $\varepsilon$ (here the topology considered on $[0,1]^{\mathbf{N}}$ is the standard product topology). Remark that for every integer $n \in \mathbf{N}$ now fixed, $A\left(z_{0}, A\left(z_{1}, \ldots, A\left(z_{n-1}, \bar{v}\left(x_{n}\right)\right)+\varepsilon_{n-1}\right)+\right.$ $\left.\left.\left.\varepsilon_{n-2}+\cdots\right)+\varepsilon_{2}\right)+\varepsilon_{1}\right)$ is an upper-semicontinuous function of the $(n-1)$-uple $\left(\varepsilon_{1}, \ldots, \varepsilon_{n-1}\right) \in \mathbf{R}^{n-1}$, from the upper semicontinuity of $A$, the upper semicontinuity of $\bar{v}$ (a consequence of Berge Maximum theorem), and from Increasing Monotonicity. Thus, $A\left(z_{0}, A\left(z_{1}, \ldots, A\left(z_{n-1}, \bar{v}\left(x_{n}\right)\right)+\varepsilon_{n-1}\right)+\varepsilon_{n-2}+\cdots\right)+$ $\left.\varepsilon_{2}\right)+\varepsilon_{1}$ ) is also an upper semicontinuous function with respect to the whole sequence $\varepsilon$ (by definition of the product topology chosen on $[0,1]^{\mathbf{N}}$ ). Passing 
to the infimum with respect to $n$, one obtains an upper-semicontinuous function of $\left(\varepsilon_{k}\right)_{k \geq 1}$ as well. Consequently, and since the feasibility contraint has a closed and compact graph, from Berge theorem, we get that $f$ is upper semicontinuous at 0 , the null sequence (in fact everywwhere).

Thus, we can recover Theorem 4.2 , which concludes that $\bar{v}^{*}\left(x_{0}\right)=\sup _{x \in \Sigma\left(x_{0}\right)} U(\underset{\sim}{x})$ is the only fixed point of the Bellman operator.

\section{REFERENCES}

[1] Alvarez F. and Stokey N., 1998, Dynamic programming with homogeneous functions, Journal of Economic Theory 82, 167-189.

[2] Becker, R. A. and Boyd, John III, 1997, Capital Theory, Equilibrum Analysis and Recursive Utility, Blackwell.

[3] Boyd, John III, 1990, Recursive utility and the Ramsey problem, Journal of Economic Theory 50, 326-345.

[4] Duran, J., 2000, On dynamic programming with unbounded returns, Economic Theory 15, 339-352.

[5] Jaśkiewicz, A., Matkowski, J. and Nowak, A.S., 2014, On variable discounting in dynamic programming: applications to resource extraction and other economic models, Annals of Operations Research, vol. 220, pp. 263278

[6] Kamihigashi T., 2014, Elementary results on solutions to the Bellman equation of dynamic programming: existence, uniqueness and convergence, Economic Theory 56, 251-273.

[7] Koopmans T., 1960, Stationary Ordinal Utility and Impatience, Econometrica 28, 287-309.

[8] Le Van C., Morhaim L., 2002, Optimal growth models with bounded or unbounded returns: a unifying approach, Journal of Economic Theory 105, 158-187. 
[9] Le Van C., Vailakis Y., 2005, Recursive utility and optimal growth with bounded or unbounded returns, Journal of Economic Theory 123 , 187209.

[10] Marinacci M., Montrucchio L., 2010, Unique solutions for stochastic recursive utilities, Journal of Economic Theory 145, 1776-1804.

[11] Martins-da-Rocha V.F., Vailakis Y., 2010, Existence and uniqueness of a fixed point for local contractions, Econometrica 78, 1127-1141

[12] Martins-da-Rocha V.F., Vailakis Y., 2012, Fixed point for local contractions: Applications to recursive utility, International Journal of Economic Theory 9, 23-33.

[13] Matkowski J., Nowak A.S., 2011, On Discounted Dynamic Programming With Unbounded Returns, Economic Theory, 46 (3), 455-474.

[14] Rincon-Zapatero J.P., Rodriguez-Palmero C., 2003, Existence and uniqueness of solutions to the Belllman equation in the unbounded case, Econometrica 71, 1519-1555.

[15] Rincon-Zapatero J.P., Rodriguez-Palmero C., 2007, Recursive utility with unbounded aggregators, Economic Theory 33, 381-390.

[16] Rincon-Zapatero J.P., Rodriguez-Palmero C., 2009, Corrigendum to Existence and uniqueness of solutions to the Belllman equation in the unbounded case, Econometrica 77, 317-318.

[17] Stokey, N. and R.E. Lucas 1984, Optimal Growth with Many Consumers, Journal of Economic Theory 32: 139-171.

[18] Stokey, N., R.E. Lucas and E. Prescott 1989, Recursive Methods in Economic Dynamics, Harvard University Press.

[19] Streufert P. A., 1990, Stationary recursive utility and dynamic programming under the assumption of biconvergence, Rev. Econ. Stud. 57, 79-97.

[20] Streufert P. A., 1992, An abstract topological approach to dynamic programming, J. Math. Econ. 21, 59-88. 
[21] Yao M., 2014, Fixed Point Theorems, Recursive Utility and the Solution to the Bellman Equation, manuscript, Keio university.

[22] Yao, M., 2016, Recursive Utility and the Solution to the Bellman Equation, manuscript, Keio university, No. DP2016-08 